\title{
Search for the flavor-changing neutral current interactions of the top quark and the Higgs boson which decays into a pair of b quarks at $\sqrt{s}=13 \mathrm{TeV}$
}

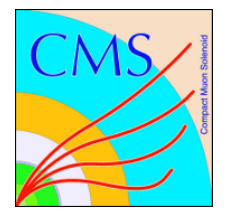

\section{The CMS collaboration}

E-mail: cms-publication-committee-chair@cern.ch

ABSTRACT: A search for flavor-changing neutral currents (FCNC) in events with the top quark and the Higgs boson is presented. The Higgs boson decay to a pair of b quarks is considered. The data sample corresponds to an integrated luminosity of $35.9 \mathrm{fb}^{-1}$ recorded by the CMS experiment at the LHC in proton-proton collisions at $\sqrt{s}=13 \mathrm{TeV}$. Two channels are considered: single top quark FCNC production in association with the Higgs boson ( $\mathrm{pp} \rightarrow \mathrm{tH}$ ), and top quark pair production with FCNC decay of the top quark $(\mathrm{t} \rightarrow$ $\mathrm{qH})$. Final states with one isolated lepton and at least three reconstructed jets, among which at least two are associated with b quarks, are studied. No significant deviation is observed from the predicted background. Observed (expected) upper limits at $95 \%$ confidence level are set on the branching fractions of top quark decays, $\mathcal{B}(\mathrm{t} \rightarrow \mathrm{uH})<0.47 \%(0.34 \%)$ and $\mathcal{B}(\mathrm{t} \rightarrow \mathrm{cH})<0.47 \%(0.44 \%)$, assuming a single nonzero FCNC coupling.

Keywords: FCNC Interaction, Hadron-Hadron scattering (experiments), Higgs physics, Top physics

ArXiv EPrint: 1712.02399 


\section{Contents}

1 Introduction 1

2 The CMS detector 2

3 Monte Carlo simulation 3

4 Event selection $\quad 4$

5 Event reconstruction and multivariate analysis 5

$\begin{array}{lll}6 & \text { Estimation of systematic uncertainties } & 6\end{array}$

$\begin{array}{lll}7 & \text { Results } & 10\end{array}$

$\begin{array}{lll}8 & \text { Summary } & 13\end{array}$

$\begin{array}{lr}\text { The CMS collaboration } & 18\end{array}$

\section{Introduction}

A recently discovered fundamental particle has properties that are consistent with the standard model (SM) predictions for the Higgs boson, H [1-4]. In the SM, flavor-changing neutral currents (FCNC) are forbidden at tree level and are strongly suppressed in loop corrections by the Glashow-Iliopoulos-Maiani (GIM) mechanism [5] with the SM branching fraction of $\mathrm{t} \rightarrow \mathrm{qH}$ predicted to be $\mathcal{O}\left(10^{-15}\right)$ [6-8]. Several extensions of the SM incorporate significantly enhanced FCNC behavior that can be directly probed at the CERN LHC [8, 9]. The FCNC processes that correspond to $\mathrm{tH}$ interactions are described by the following effective Lagrangian:

$$
\mathcal{L}=\sum_{\mathrm{q}=\mathrm{u}, \mathrm{c}} \frac{g}{\sqrt{2}} \overline{\mathrm{t}} \kappa_{\mathrm{Hqt}}\left(f_{\mathrm{Hq}}^{\mathrm{L}} P_{\mathrm{L}}+f_{\mathrm{Hq}}^{\mathrm{R}} P_{\mathrm{R}}\right) \mathrm{q} \mathrm{H}+\text { h.c. },
$$

where $g$ is the weak coupling constant, $P_{\mathrm{L}}$ and $P_{\mathrm{R}}$ are chirality projectors in spin space, $\kappa_{\mathrm{Hqt}}$ is the effective coupling, $f_{\mathrm{Hq}}^{\mathrm{L}}$ and $f_{\mathrm{Hq}}^{\mathrm{R}}$ are left- and right-handed complex chiral parameters with a unitarity constraint of $\left|f_{\mathrm{Hq}}^{\mathrm{L}}\right|^{2}+\left|f_{\mathrm{Hq}}^{\mathrm{R}}\right|^{2}=1$. The tH FCNC interaction is studied in this analysis in two channels: the associated production of a single top quark with the Higgs boson (ST), as well as in FCNC decays of top quarks in t $\bar{t}$ semileptonic events (TT). In this analysis, $\mathrm{H} \rightarrow \mathrm{b} \bar{b}$ decays are considered. This is the first time that the analysis of the ST mode is presented. Representative Feynman diagrams of the studied processes are shown in figure 1. 

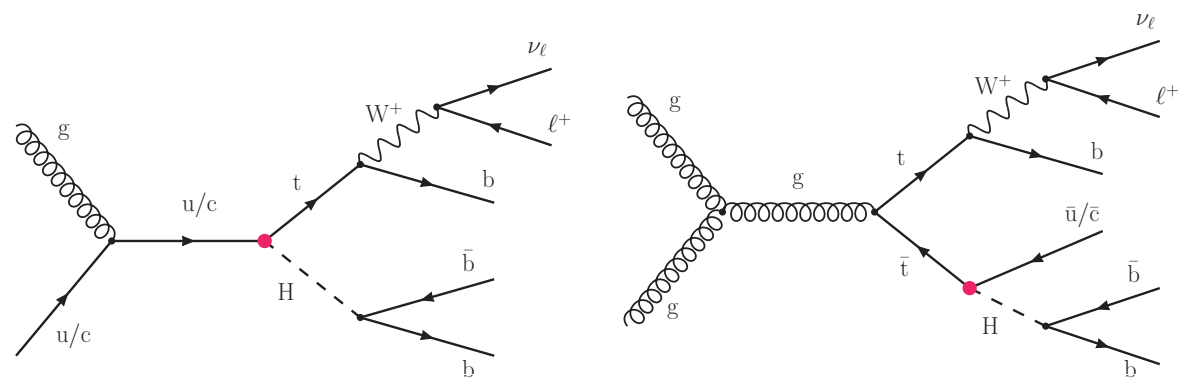

Figure 1. Representative Feynman diagrams for FCNC tH processes: associated production of the

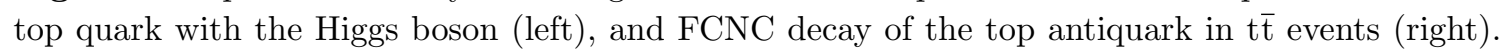
The FCNC vertex is indicated by the bullet.

Earlier analyses by the ATLAS [10, 11] and CMS [12] Collaborations have probed $\kappa_{\text {Hqt }}$ in top quark decays in $t \bar{t}$ events. The ATLAS search at center-of-mass energy of $13 \mathrm{TeV}$ investigated the $\mathrm{t} \rightarrow \mathrm{qH}$ decay with the Higgs boson decaying to two photons to set observed (expected) upper limits at $95 \%$ confidence level (CL) on the branching fractions $\mathcal{B}(\mathrm{t} \rightarrow \mathrm{uH})$ and $\mathcal{B}(\mathrm{t} \rightarrow \mathrm{cH})$ of $0.24 \%(0.17 \%)$ and $0.22 \%(0.16 \%)$, respectively [11]. The CMS analysis at $\sqrt{s}=8 \mathrm{TeV}$ utilized the Higgs boson decays into either boson or fermion pairs to set observed (expected) upper limits of $0.55 \%(0.40 \%)$ and $0.40 \%(0.43 \%)$ on $\mathcal{B}(\mathrm{t} \rightarrow \mathrm{uH})$ and $\mathcal{B}(\mathrm{t} \rightarrow \mathrm{cH})$, respectively [12].

For the signal processes, we consider the cross section times branching fraction with a specific signature for single top quark $\mathrm{t}\left(\rightarrow \ell^{+} \nu \mathrm{b}\right) \mathrm{H}(\rightarrow \mathrm{b} \overline{\mathrm{b}})$ and pair production $\mathrm{t}(\rightarrow$ $\left.\ell^{+} \nu \mathrm{b}\right) \overline{\mathrm{t}}(\rightarrow \overline{\mathrm{u}} / \overline{\mathrm{c}} \mathrm{H}(\rightarrow \mathrm{b} \overline{\mathrm{b}}))$, with $\ell=\mathrm{e}, \mu$, or $\tau$. The analysis also considers the chargeconjugate process. The predicted cross section at $13 \mathrm{TeV}$ for single top quark and antiquark FCNC production in association with the Higgs boson under the assumption of coupling strengths $\kappa_{\mathrm{Hut}}=1, \kappa_{\mathrm{Hct}}=0\left(\kappa_{\mathrm{Hct}}=1, \kappa_{\mathrm{Hut}}=0\right)$ is $13.8(1.90) \mathrm{pb}$, where the cross section calculation is based on the leading order (LO) set of NNPDF 2.3 parton distribution functions (PDFs) [13]. In the case of the production of $t \bar{t}$ semileptonic events with top quark FCNC decay, the predicted cross section is $37.0 \mathrm{pb}$ and is independent of the type of the coupling. By exploiting a simultaneous analysis of both the TT and ST processes, an improved sensitivity to $\kappa_{\text {Hut }}$ can be achieved, as the ST production via the up quark is enhanced by the proton PDFs.

This analysis uses data that correspond to an integrated luminosity of $35.9 \mathrm{fb}^{-1}$ [14] recorded in 2016 by the CMS experiment at the LHC in proton-proton (pp) collisions at $\sqrt{s}=13 \mathrm{TeV}$. Events with exactly one isolated lepton (electron or muon) and with at least three jets, among which at least two are associated with b quarks, are considered.

\section{The CMS detector}

The central feature of the CMS detector is a superconducting solenoid of $6 \mathrm{~m}$ internal diameter, providing a magnetic field of $3.8 \mathrm{~T}$. Within the solenoid volume are a silicon pixel and strip tracker, a lead tungstate crystal electromagnetic calorimeter (ECAL), and a brass and scintillator hadron calorimeter (HCAL), each composed of a barrel and two 
endcap sections. Forward calorimeters extend the pseudorapidity coverage provided by the barrel and endcap detectors. Muons are measured in gas-ionization detectors embedded in the steel flux-return yoke outside the solenoid. A more detailed description of the CMS detector, together with a definition of the coordinate system used and the relevant kinematic variables, can be found in ref. [15].

\section{Monte Carlo simulation}

The generation of simulated signal events is done at LO with MADGRAPH5_amC@NLO 2.3.3 [16, 17]. Up to two additional partons are simulated by the Monte Carlo (MC) generator in the initial hard process for the top quark pair production mode. The MLM [18] matching scheme is used to match additional partons in the matrix-element calculations to the parton-shower description. No additional partons are included in the generation of events for the single top quark production process, as such inclusion would contain contributions from the top quark pair production process. A systematic variation in the normalization of the single top production process by $10 \%$ is considered in order to account for the differences in the generation of additional radiation of the two signal production modes. The Lagrangian terms from eq. (1.1) are implemented by means of the FEYNRULES package [19] using the universal FeynRules output format [20]. The complex chiral parameters are fixed to $f_{\mathrm{Hq}}^{\mathrm{R}}=1$ and $f_{\mathrm{Hq}}^{\mathrm{L}}=0$.

The SM top quark pair production is the dominant background process and is simulated to next-to-leading order (NLO) using POWHEG v2 [21-24]. The predicted cross section for this process is $832{ }_{-29}^{+20}$ (scale) $\pm 35(\mathrm{PDF}) \mathrm{pb}$, as calculated with the ToP ++2.0 program at next-to-next-to-leading order (NNLO), including soft-gluon resummation to next-tonext-to-leading-log order (see ref. [25] and references therein), and assuming a top quark mass of $m_{\mathrm{t}}=172.5 \mathrm{GeV}$. Two systematic uncertainties that are shown in the prediction are considered. These are independent variations of the factorization and renormalization scales, $\mu_{\mathrm{F}}$ and $\mu_{\mathrm{R}}$, and variations of the $\mathrm{PDF}$ and $\alpha_{s}$.

Single top quark production in the $t$ channel is simulated with POWHEG v2 in the four-flavour scheme, while events for single top quark production in association with $\mathrm{W}$ bosons are generated with POWHEG v1 in the five-flavour scheme $(5 \mathrm{FS})$. The predicted NLO cross sections are $217_{-8}^{+9}[26,27]$ and $71.7 \pm 3.9 \mathrm{pb}$ [28], respectively. Single top quark production in the $s$ channel is done at NLO with the MADGRAPH5_aMC@NLO generator in $5 \mathrm{FS}$ with a predicted cross section of $10.3 \pm 0.4 \mathrm{pb}$. The uncertainties in the quoted cross sections correspond to independent variations of $\mu_{\mathrm{F}}$ and $\mu_{\mathrm{R}}$, as well as to variations of the PDF and $\alpha_{s}$. Small contributions to the overall predicted background arise from several additional processes: $\mathrm{W}$ boson production and the associated production of $\mathrm{t} \overline{\mathrm{t}}$ with W and Z, both generated with MADGraph5_amC@NLO, and from Drell-Yan and the associated production of $t \bar{t}$ with a Higgs boson generated with the MADGRAPH5_aMC@NLO and POWHEG v1 [29], respectively.

In the simulation of signal and background processes, the initial- and final-state radiation (ISR and FSR), as well as the fragmentation and hadronization of quarks, are

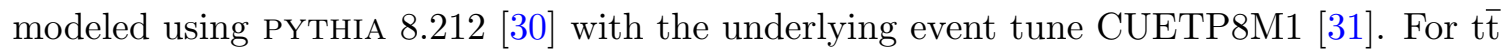


generation, the first emission is done at the matrix-element level with POWHEG v2. Generation of $t \bar{t}$ and single top quark production in the $t$ channel uses the underlying event tune CUETP8M2T4 [32]. In the generation of all background processes the NNPDF3.0 $\mathrm{PDF}[33]$ set is used.

The detector response is simulated using GEANT4 v9.4 [34]. In order to model the effect of multiple interactions per event crossing (pileup), generated minimum bias events were added to the simulated data. The number of extra multiple interactions were matched to agree with the rate observed in data. The number of pileup interactions in data is estimated from the measured bunch-to-bunch instantaneous luminosity and the total inelastic cross section $(69.2 \mathrm{mb})[14]$.

\section{Event selection}

The particle-flow (PF) algorithm [35] reconstructs and identifies each individual particle with an optimized combination of information from the various elements of the CMS detector. The energy of photons is directly obtained from the ECAL measurement, corrected for zero-suppression effects. The energy of electrons is determined from a combination of the electron momentum at the primary interaction vertex as determined by the tracker, the energy of the corresponding ECAL cluster, and the energy sum of all bremsstrahlung photons spatially compatible with originating from the electron track. The momentum of muons is obtained from the curvature of the corresponding track. The energy of charged hadrons is determined from a combination of their momentum measured in the tracker and the matching ECAL and HCAL energy deposits, corrected for zero-suppression effects and for the response function of the calorimeters to hadronic showers. Finally, the energy of neutral hadrons is obtained from the corresponding corrected ECAL and HCAL energy.

Jets are reconstructed by clustering PF candidates using the anti- $k_{\mathrm{T}}$ algorithm $[36,37]$ with a distance parameter of 0.4. The jet momentum is determined as the vectorial sum of all particle momenta in the jet, and is found from simulation to be within 5 to $10 \%$ of the true momentum over the whole transverse momentum $\left(p_{\mathrm{T}}\right)$ spectrum and detector acceptance [38]. An offset correction is applied to jet energies to take into account the contribution from pileup. Jet energy corrections are derived from simulation and are confirmed with in situ measurements of the energy balance in dijet, multijet, $\gamma+$ jet, and leptonic $\mathrm{Z}+$ jet events. Additional selection criteria are applied to each event to remove spurious jet-like features originating from isolated noise patterns in certain HCAL regions. The missing transverse momentum $\left(\vec{p}_{\mathrm{T}}^{\text {miss }}\right)$ in an event is defined as the magnitude of the transverse projection of the vector sum of the momenta of all reconstructed PF candidates in an event.

The reconstructed vertex with the largest value of summed physics-object $p_{\mathrm{T}}^{2}$ is taken to be the primary pp interaction vertex. The physics objects are the jets, clustered using the jet finding algorithm $[36,37]$ with the tracks assigned to the vertex as inputs, and the associated $\vec{p}_{\mathrm{T}}^{\text {miss }}$, taken as the negative vector $p_{\mathrm{T}}$ sum of those jets, to represent the neutral particles. 
This analysis selects events with exactly one isolated lepton (electron or muon). Events with one electron (muon) are recorded using a trigger that required at least one electron (muon) with $p_{\mathrm{T}}>32(24) \mathrm{GeV}$ selected within the detector acceptance $(|\eta|<2.1)$. Electron (muon) candidates are selected offline with $|\eta|<2.1$ with $p_{\mathrm{T}}>35(30) \mathrm{GeV}$. Electrons that are reconstructed in the transition region between the barrel and endcap regions of the ECAL, $1.44<|\eta|<1.57$, are removed. Leptons are required to be isolated in terms of a relative isolation variable, $I_{\text {rel }}$. This variable is defined as the ratio of the scalar $p_{\mathrm{T}}$ sum of photons, charged hadrons, and neutral hadrons within a cone of angular radius $\Delta R=\sqrt{(\Delta \eta)^{2}+(\Delta \phi)^{2}}=0.3(0.4)$ of the reconstructed lepton candidate, where $\phi$ is azimuthal angle in radians, to the lepton $p_{\mathrm{T}}$. This isolation variable only includes the charged hadrons that emerge from the same vertex as the selected lepton and is corrected for energy deposits from neutral particles produced in pileup interactions. For electron (muon) candidates, $I_{\text {rel }}$ must be less than $0.06(0.15)$. In order to suppress background processes with multilepton final states, events with additional leptons passing the looser isolation requirement of $I_{\text {rel }}<0.25$ and $p_{\mathrm{T}}>10 \mathrm{GeV}$ are rejected.

At least three jets are required to be present in the event with $p_{\mathrm{T}}>30 \mathrm{GeV}$ and $|\eta|<2.4$. As signal events contain three b quarks produced in the final state at the tree level, we require at least two jets are identified as b quark jets by the combined secondary vertex v2 (CSVv2) b tagging algorithm [39]. This requirement corresponds to the selection of jets with the CSVv2 discriminant value greater than 0.85 , and provides a $b$ jet efficiency of $\approx 70 \%$, with a misidentification rate for $\mathrm{c}$ jets and jets originating from light quarks and gluons of $\approx 10 \%$ and $\approx 1 \%$, respectively.

\section{Event reconstruction and multivariate analysis}

In order to optimize sensitivity to the signal event selection, events are split into five categories based on the total number of reconstructed jets and on the number of b-tagged jets. Categories with exactly three jets of which two or three are identified as b jets are denoted as b2j3 and b3j3, respectively. Similarly, categories with at least four jets of which two, three, or four are identified as b jets are specified as $\mathrm{b} 2 \mathrm{j} 4$, b3j4, and $\mathrm{b} 4 \mathrm{j} 4$, respectively The longitudinal momentum of the neutrino is determined by assigning $\vec{p}_{\mathrm{T}}^{\text {miss }}$ to the neutrino, and constraining the $\ell \nu$ mass to the known mass of the $\mathrm{W}$ boson. With the use of the energy and momenta of all particles, a full kinematic reconstruction of the event is performed for several signal and background hypotheses: ST, TT, and background t $\overline{\mathrm{t}}$ events, where one of the top quarks decays semileptonically, and the other one hadronically. The reconstruction is performed for all possible permutations of the b-tagged jets to be associated with the decay products of the Higgs boson or the top quark, and both solutions for the longitudinal momentum of the neutrino are considered. The reconstructed kinematic variables for each permutation are then fed into a multivariate analysis that uses a boosted decision tree (BDT) [40] approach, as implemented in the toolkit for multivariate analysis TMVA [41]. The input BDT variables that are used for the ST and TT hypotheses correspond to the reconstructed invariant mass of two b jets associated with the Higgs boson decay, the reconstructed invariant mass of a b jet $\left(m_{\mathrm{b} \overline{\mathrm{b}}}\right)$, lepton and neutrino associated 
with the top quark decay $\left(m\left(\mathrm{t}^{\ell}\right)\right)$, its transverse momentum $\left(p_{\mathrm{T}}\left(\mathrm{t}^{\ell}\right)\right), \Delta R$ between the reconstructed Higgs boson and the top quark. In case of the hypothesis of the background $t \bar{t}$ events the following variables are used: $m\left(\mathrm{t}^{\ell}\right), m\left(\mathrm{t}^{\mathrm{h}}\right), \Delta R\left(\mathrm{t}^{\ell}, \mathrm{t}^{\mathrm{h}}\right)$, and $p_{\mathrm{T}}\left(\mathrm{t}^{\ell}\right)$, where $\mathrm{t}^{\mathrm{h}}$ corresponds to the reconstructed top quark hadronic decay from one b-tagged and two non b-tagged jets. The BDT classifier is trained to distinguish the correct from the wrong $\mathrm{b}$ jet assignments. The training and validation of the BDT is performed on statistically independent simulated samples. All reconstructed b jets in the event are considered, and the permutation with the highest BDT score is chosen as the correct one. The measured algorithm efficiency for correct assignment of the b-tagged jets to the jets reconstructed at generator level after applying the analysis selection criteria is $\approx 75 \%$.

Kinematic variables from the event reconstruction are used to construct several BDTs to suppress backgrounds. The BDTs are trained for each jet multiplicity category to identify signal events that are generated either for $\kappa_{\text {Hut }}$ (Hut) or $\kappa_{\text {Hct }}$ (Hct) coupling against the sum of all background events. Separate trainings of the BDT for Hut and Hct are done in order to take into account the differences in kinematic properties of the reconstructed objects in the ST production mode, as well as the differences in the measured b tagging efficiencies for a charm and an up quark in the TT production channel. The most important variables that discriminate between signal and background events are: the charge of the lepton (considered only for the BDT that uses Hut signal events), the CSVv2 discriminant value of the $\mathrm{b}$ jet with the lowest $p_{\mathrm{T}}$ from the Higgs boson decay, $m_{\mathrm{b} \overline{\mathrm{b}}}$, and the output discriminant value of the BDT used in the $\mathrm{b}$ jet assignment procedure. Distributions for these variables in data and $\mathrm{MC}$ simulation in the b3j3 category are presented in figure 2. The b4j4 category is not considered for Hut due to negligible improvement in the final sensitivity.

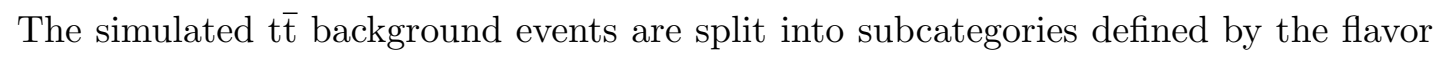
of additional particle-level jets produced in association with the top quark pair. These classes are referred to as $t \bar{t}+b \bar{b}, t \bar{t}+c \bar{c}$, and $t \bar{t}+l f$, (where lf stands for light flavor). The $t \bar{t}+$ lf category includes events where no additional pair of $b$ or $c$ jets occurs. The other background processes are summed up and shown together in the prediction.

The final observable used to extract signal events is defined as the BDT score distribution in each jet category corresponding to either Hut or Hct signal training. Figures 3 and 4 show the comparison between data and simulation for this observable after the fit to data with all background processes constrained to the SM expectation.

\section{Estimation of systematic uncertainties}

Sources of systematic uncertainty that affect both the normalization and shape of the predicted signal and background events are considered in the analysis. All systematic uncertainties are treated as nuisance parameters in the derivation of the exclusion limit.

The dominant systematic uncertainty arises from the application of the $b$ tagging requirement. The shape of the $\mathrm{CSVv} 2$ discriminant, the $\mathrm{b}$ tagging efficiency, and the misidentification rate in simulation are corrected to reproduce the data distributions [39]. The uncertainties that are associated with these correction factors are the statistical uncer- 

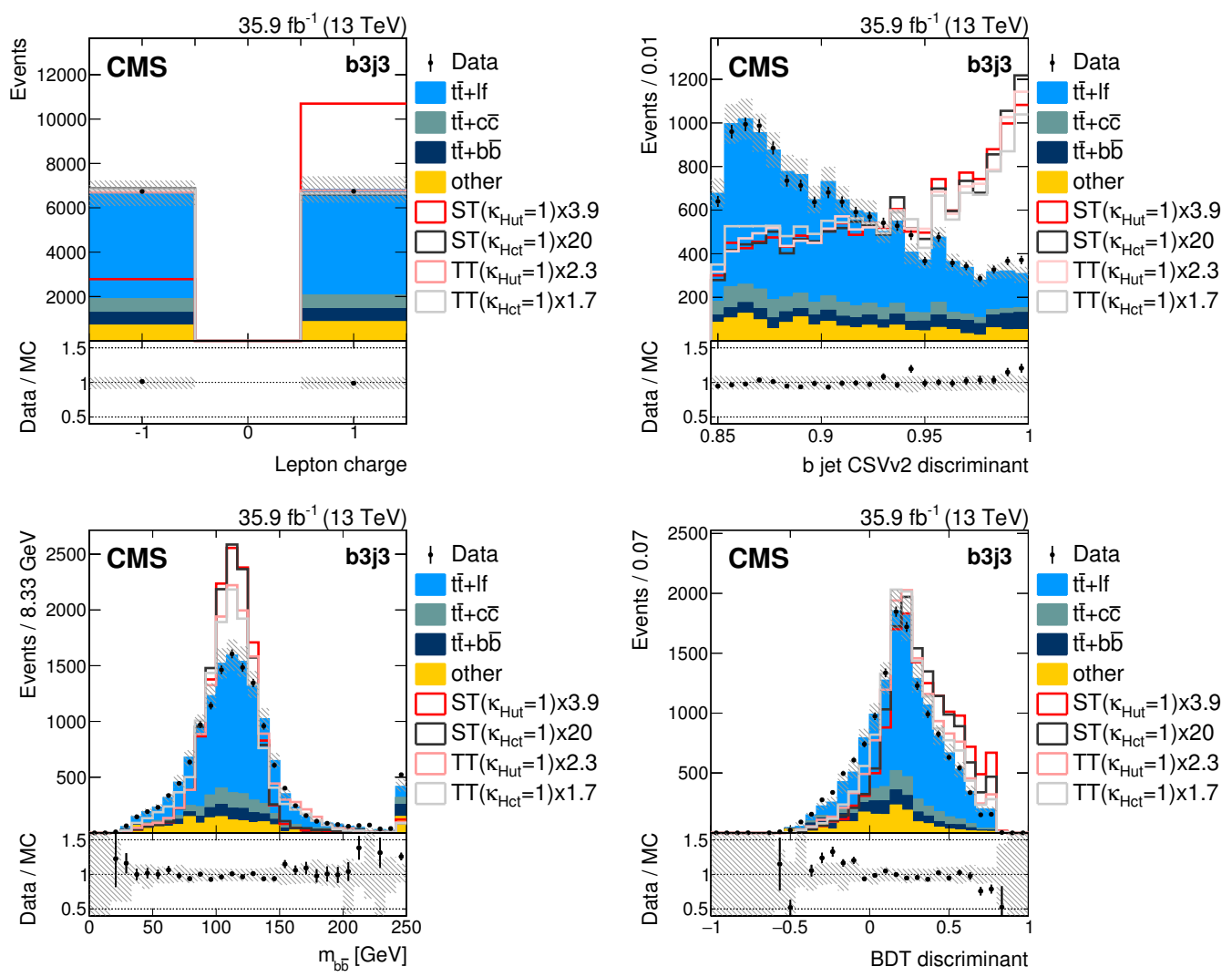

Figure 2. Comparison between data and simulation for the most discriminating BDT input variables in the b3j3 category: lepton charge (upper left), CSVv2 discriminant value for one of the reconstructed $\mathrm{b}$ jets assigned to Higgs boson decay (upper right), reconstructed invariant mass of two $b$ jets associated with the Higgs boson decay (lower left), and the maximum BDT discriminant value from the $\mathrm{b}$ jet assignment procedure (lower right). The last bin in the distribution for the reconstructed mass of the Higgs boson includes the overflows. The shaded area corresponds to the total uncertainty in the predicted background. The data-to-simulation ratio is also shown. The distributions for the signal processes are normalized to the total number of events in the predicted background to ease the comparison of the shapes of the distributions.

tainty due to the limited data sample from which the correction factors were derived, and the systematic uncertainty arising from the purity estimate of the sample as predicted by simulation. The overall effect of this systematic uncertainty leads to a variation of $\approx 8-30 \%$ in simulated event yields, with the largest effect observed in event categories with a large number of b-tagged jets.

The uncertainty associated with the choice of renormalization and factorization scales in the matrix element is estimated by changing each scale separately by a factor of $1 / 2$ and 2. To estimate the systematic uncertainty at the parton-shower level, several special simulated samples of events are considered, where the scales used to determine the ISR and FSR emissions are varied. The uncertainty associated with the choice of PDF is estimated by using several PDFs and assigning the maximum differences as the quoted uncertainty, following the PDF4LHC prescription with the MSTW2008 68\% CL NNLO, 

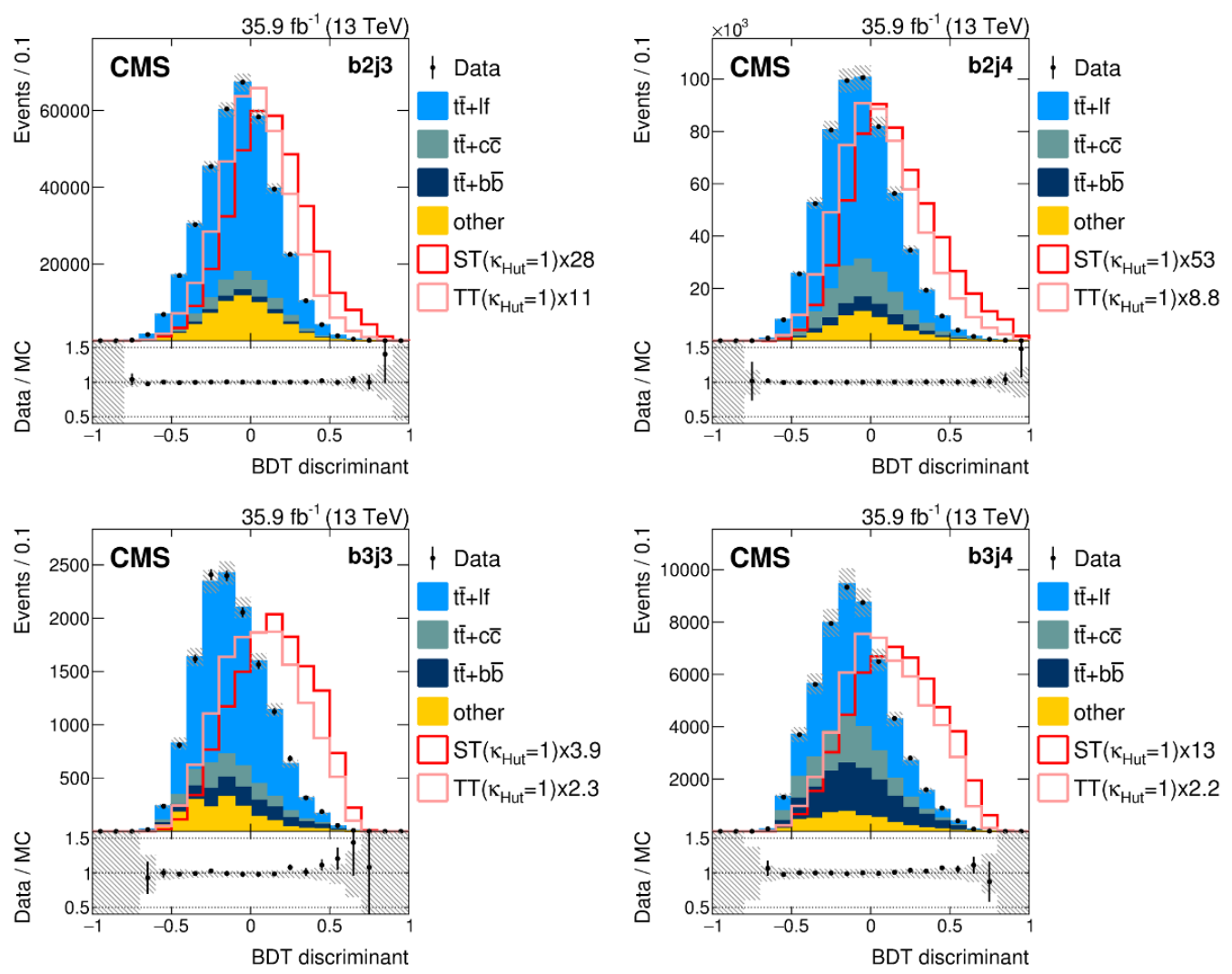

Figure 3. The BDT discriminant distributions for different jet categories for Hut training after the fit to data. All background processes are constrained to the SM expectation in the fit. The shaded area corresponds to the total uncertainty in the predicted background. The data-to-simulation ratio is also shown. The distributions for the signal processes are normalized to the total number of events in the predicted background to ease the comparison of the shapes of the distributions.

CT10 NNLO, and NNPDF2.3 5f FFN PDF sets (see ref. [42] and references therein, as well as refs. $[13,43,44])$. The overall uncertainty associated with the simulation of the background processes contributes up to $\approx 20 \%$ in the variation of event yields.

Following the prescription in POWHEG [32], the matching of the high- $p_{\mathrm{T}}$ partons, from matrix-element calculations and parton-shower emission, is regulated by damping the emission by the factor $m_{\mathrm{t}}^{2} /\left(p_{\mathrm{T}}^{2}+m_{\mathrm{t}}^{2}\right)$. Additional simulated samples for $\mathrm{t} \overline{\mathrm{t}}$ are used that correspond to the variation of this factor within the considered uncertainty. For the t $\bar{t}$ and single top quark $t$-channel simulated samples the additional systematic uncertainties associated with the amount of multiparton interactions and color reconnection $[45,46]$ are considered. These uncertainties were determined by varying them according to the uncertainties reported for the underlying event tune CUETP8M2T4, and lead to a systematic effect of $\approx 1-5 \%$.

The uncertainty associated with the calibration of the jet energy scale and the jet energy resolution contributes up to $\approx 8 \%$ in the variation of the final event yields [47]. The identification, isolation, and trigger efficiency correction uncertainties for reconstructed 

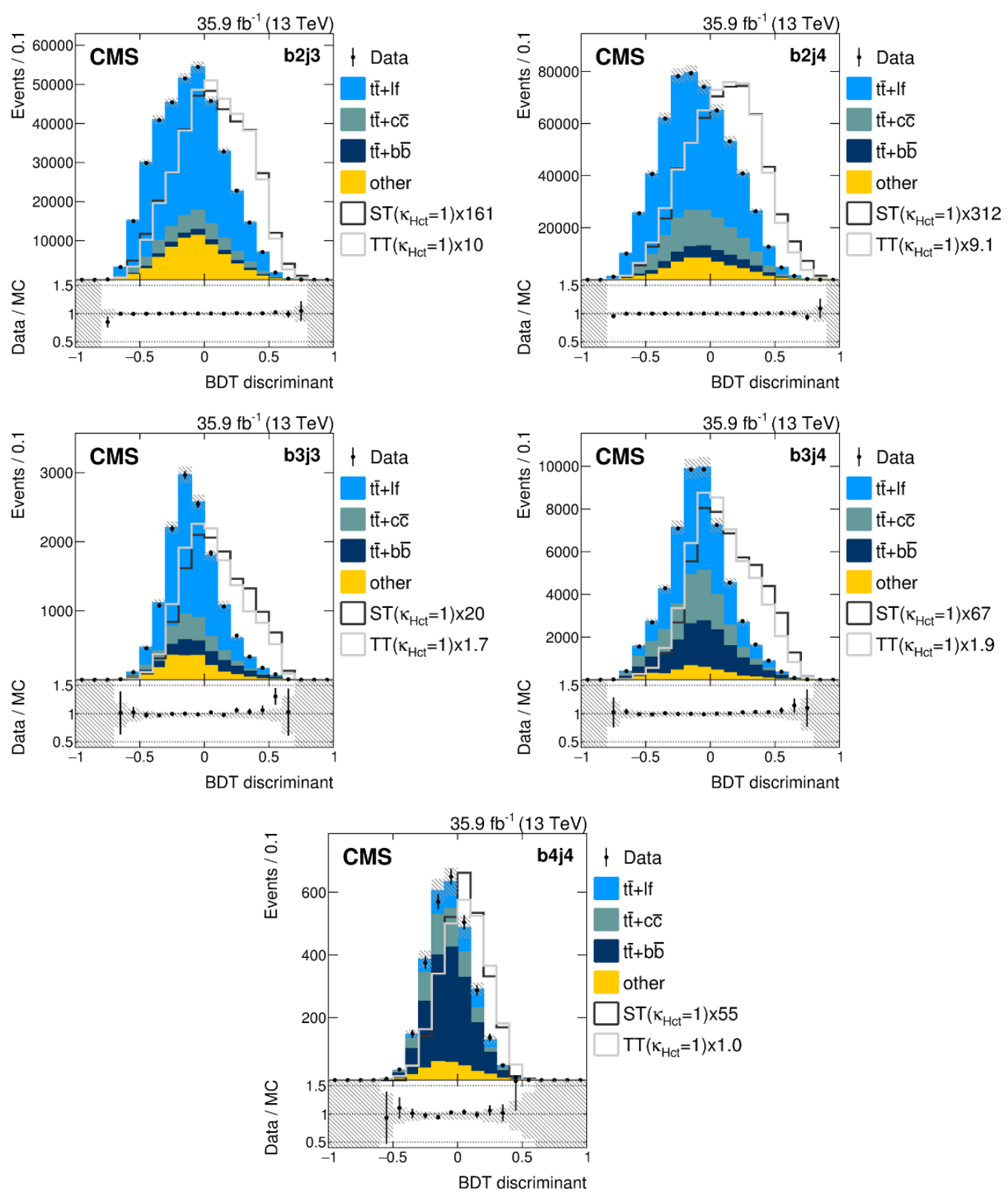

Figure 4. The BDT discriminant distributions for different jet categories for Hct training after the fit to data. All background processes are constrained to the SM expectation in the fit. The shaded area corresponds to the total uncertainty in the predicted background. The data-to-simulation ratio is also shown. The distributions for the signal processes are normalized to the total number of events in the predicted background to ease the comparison of the shapes of the distributions. 


\begin{tabular}{|c|cccc|}
\hline & $\mathrm{b} 2 \mathrm{j} 3$ & $\mathrm{~b} 2 \mathrm{j} 4$ & $\mathrm{~b} 3 \mathrm{j} 3$ & $\mathrm{~b} 3 \mathrm{j} 4$ \\
\hline Data & 365890 & 575500 & 13481 & 53352 \\
\hline $\mathrm{t} \overline{\mathrm{t}}+\mathrm{b} \overline{\mathrm{b}}$ & $8880 \pm 3641$ & $30157 \pm 5127$ & $1214 \pm 510$ & $11668 \pm 1750$ \\
$\mathrm{t} \overline{\mathrm{t}}+\mathrm{c} \overline{\mathrm{c}}$ & $26035 \pm 11195$ & $81959 \pm 18031$ & $1281 \pm 576$ & $9753 \pm 2243$ \\
$\mathrm{t} \overline{\mathrm{t}}+\mathrm{lf}$ & $270989 \pm 13820$ & $410028 \pm 16401$ & $9104 \pm 674$ & $27079 \pm 1733$ \\
other & $58991 \pm 6489$ & $51845 \pm 6221$ & $1616 \pm 356$ & $4269 \pm 768$ \\
\hline Total & $364895 \pm 22623$ & $573989 \pm 25256$ & $13215 \pm 1255$ & $52769 \pm 3430$ \\
\hline
\end{tabular}

Table 1. Number of events in each category together with its total relative uncertainty as obtained from the fit to data for Hut.

leptons contribute up to $0.5 \%$ of the total uncertainty in the predicted yield. An uncertainty of $2.5 \%$ is assigned to the measured integrated luminosity value of the considered data sample [14].

The number of simulated pileup events is corrected to match the measured number of events in data. The uncertainty on the total inelastic cross section is taken as $4.6 \%$. Its overall contribution to the total systematic uncertainty is found to be negligible.

The $p_{\mathrm{T}}$ spectrum of individual top quarks in data is found to be softer than predicted by the simulation. A correction for the top quark $p_{\mathrm{T}}$ spectrum in simulation is applied and the difference between the initial and the corrected shapes is taken as an additional systematic uncertainty [48]. This uncertainty also has a negligible impact on the final distributions.

Additionally, a systematic uncertainty of $50 \%$ in the predicted cross sections for $\mathrm{t} \overline{\mathrm{t}}+\mathrm{b} \overline{\mathrm{b}}$ and $\mathrm{t} \overline{\mathrm{t}}+\mathrm{c} \overline{\mathrm{c}}$ processes is assumed $[49,50]$.

\section{Results}

A comparison between the number of selected events in data and simulation is shown in tables 1 and 2. A 95\% CL upper limit is computed for the production cross section of tH FCNC events times branching fractions of top quark semileptonic decay and Higgs boson decay to $\mathrm{b}$ quarks that uses the asymptotic approximation of the $\mathrm{CL}_{\mathrm{s}}$ method $[51,52]$. The profile likelihood ratio test statistic [53] is defined as $q(\mu)=-2 \ln \left(L\left(\mu, \hat{\theta}_{\mu}\right) / L(\hat{\mu}, \hat{\theta})\right)$, where $L$ is a binned likelihood function, $\mu$ is a signal strength modifier, $\theta$ is a set of nuisance parameters, $\hat{\theta}_{\mu}$ is a set of nuisance parameters that maximize $L$ for a given $\mu$, $\hat{\mu}$ and $\hat{\theta}$ are the values of the corresponding parameters which simultaneously maximize $L$. Uncertainties due to normalization are included through nuisance parameters with log-normal prior distributions, while shape uncertainties are included with Gaussian prior distributions. The expected and observed 95\% CL upper limits are derived on the signal production cross section separately for each event category, as well as for their combination (figure 5). In the latter case, a simultaneous binned maximum-likelihood fit to all categories is performed. The fit takes into account the statistical and systematic uncertainties in the final BDT score distributions in each jet category.

The resultant observed (expected) 95\% CL exclusion limits on top quark FCNC decay branching fractions are $\mathcal{B}(\mathrm{t} \rightarrow \mathrm{uH})<0.47 \%(0.34 \%)$ and $\mathcal{B}(\mathrm{t} \rightarrow \mathrm{cH})<0.47 \%(0.44 \%)$. 

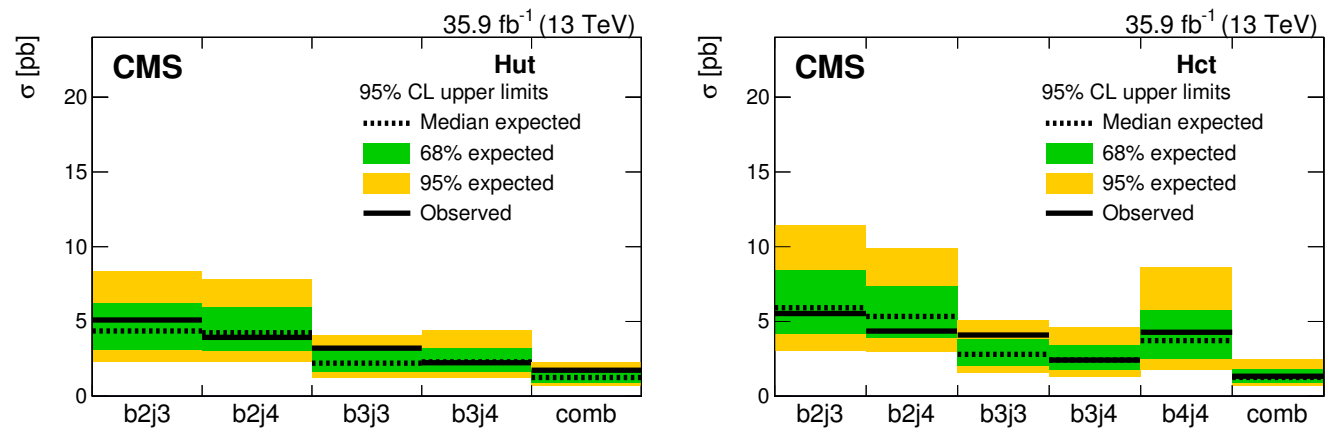

Figure 5. Excluded signal cross section at 95\% CL per event category for Hut (left) and Hct (right).

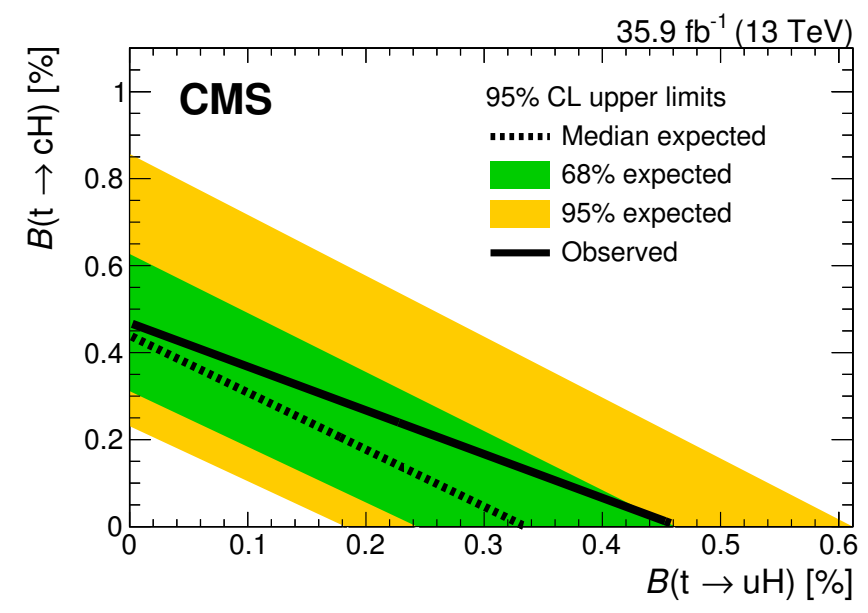

Figure 6. Upper limits on $\mathcal{B}(\mathrm{t} \rightarrow \mathrm{uH})$ and $\mathcal{B}(\mathrm{t} \rightarrow \mathrm{cH})$ at $95 \% \mathrm{CL}$.

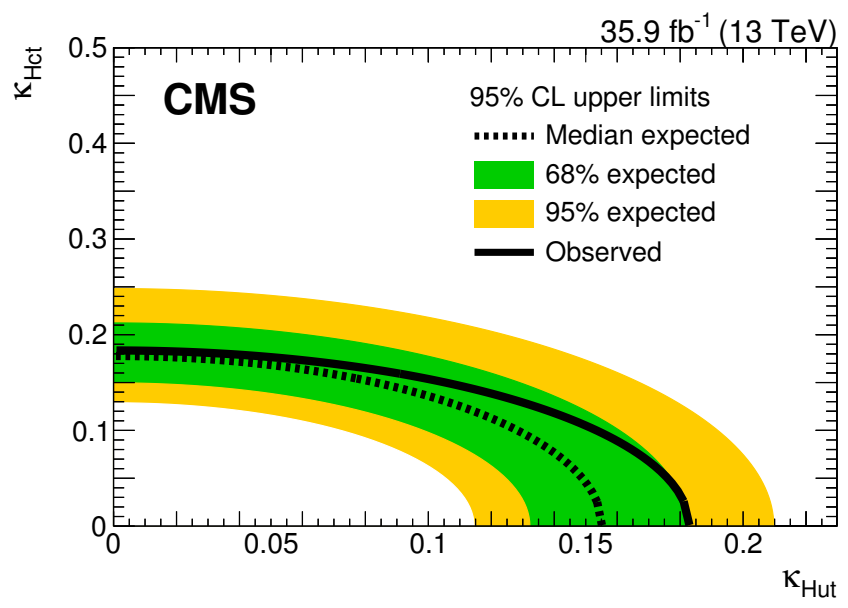

Figure 7. Upper limits on $\kappa_{\mathrm{Hut}}$ and $\kappa_{\mathrm{Hct}}$ at $95 \% \mathrm{CL}$. 


\begin{tabular}{|c|ccccc|}
\hline & $\mathrm{b} 2 \mathrm{j} 3$ & $\mathrm{~b} 2 \mathrm{j} 4$ & $\mathrm{~b} 3 \mathrm{j} 3$ & $\mathrm{~b} 3 \mathrm{j} 4$ & $\mathrm{~b} 4 \mathrm{j} 4$ \\
\hline Data & 365890 & 575500 & 13481 & 53352 & 2764 \\
\hline $\mathrm{t} \overline{\mathrm{t}}+\mathrm{b} \overline{\mathrm{b}}$ & $10176 \pm 1933$ & $34174 \pm 3759$ & $1367 \pm 273$ & $12897 \pm 1058$ & $1517 \pm 129$ \\
$\mathrm{t} \overline{\mathrm{t}}+\mathrm{c} \overline{\mathrm{c}}$ & $33210 \pm 11956$ & $102186 \pm 15328$ & $1674 \pm 619$ & $12280 \pm 1842$ & $521 \pm 104$ \\
$\mathrm{t} \overline{\mathrm{t}}+\mathrm{lf}$ & $258679 \pm 8795$ & $385395 \pm 10791$ & $8349 \pm 451$ & $24083 \pm 1132$ & $383 \pm 69$ \\
other & $62887 \pm 5723$ & $52134 \pm 6256$ & $1742 \pm 401$ & $3513 \pm 562$ & $262 \pm 50$ \\
\hline Total & $364952 \pm 16788$ & $573889 \pm 18364$ & $13132 \pm 959$ & $52773 \pm 2322$ & $2682 \pm 185$ \\
\hline
\end{tabular}

Table 2. Number of events in each category together with its total relative uncertainty as obtained from the fit to data for Hct.
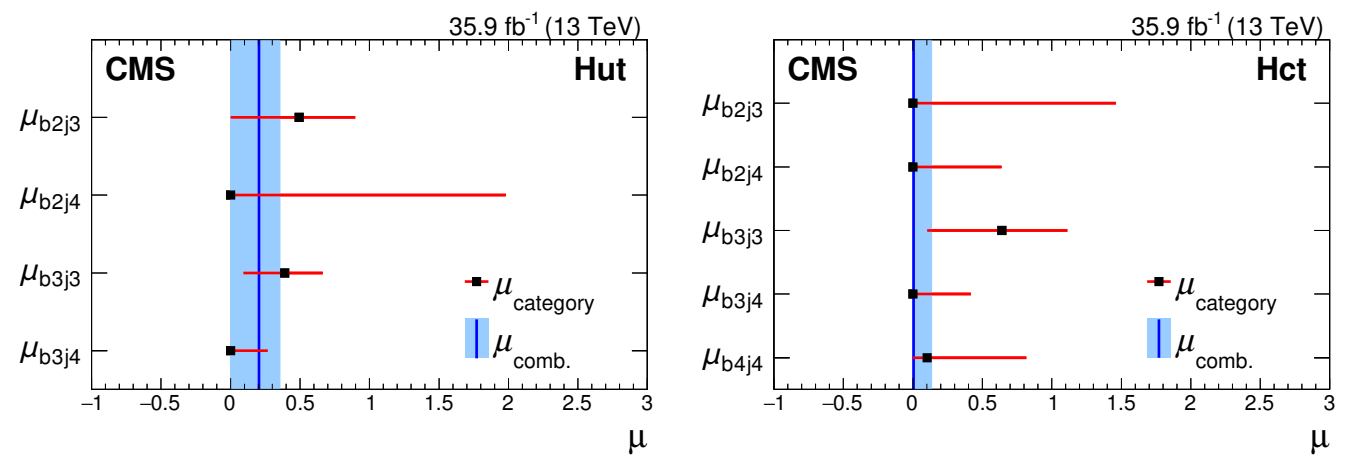

Figure 8. The best fit signal strength $(\mu)$ for Hut (left) and Hct (right), which is restricted to positive values in the fit.

These upper limits on the branching fractions can be translated into upper limits on the coupling strengths using the relations:

$$
\begin{gathered}
\kappa_{\mathrm{Hut}}^{2}=\mathcal{B}(\mathrm{t} \rightarrow \mathrm{uH}) \frac{\Gamma_{\mathrm{t}}}{\Gamma_{\mathrm{Hut}}}, \\
\kappa_{\mathrm{Hct}}^{2}=\mathcal{B}(\mathrm{t} \rightarrow \mathrm{cH}) \frac{\Gamma_{\mathrm{t}}}{\Gamma_{\mathrm{Hct}}},
\end{gathered}
$$

where the total top quark width is $\Gamma_{\mathrm{t}}=1.32 \mathrm{GeV}$ [54], and the partial width for the FCNC decay process of the top quark is $\Gamma_{\mathrm{Hut}}=\Gamma_{\mathrm{Hct}}=0.184 \mathrm{GeV}$ for $\kappa_{\mathrm{Hut}}=\kappa_{\mathrm{Hct}}=1$. The resultant limits on the coupling strengths are $\kappa_{\mathrm{Hut}}<0.18(0.16)$ and $\kappa_{\mathrm{Hct}}<0.18(0.18)$. These limits are very competitive to the CMS result with the combination of various channels at $8 \mathrm{TeV}$ [12], while the ATLAS result with the analysis of the $\mathrm{H} \rightarrow \gamma \gamma$ decay at $13 \mathrm{TeV}$ [11] represents the best limits to date. The measured one-dimensional exclusion limits are also interpreted for the scenario of the non-vanishing FCNC couplings via a linear interpolation. The results for two-dimensional limits on top quark FCNC decay branching fractions and coupling strengths are presented in figure 6 and 7 , respectively. We define a signal strength parameter as $\mu=\sigma / \sigma_{\text {sig }}$, where $\sigma$ is the cross section excluded at $95 \%$ CL and $\sigma_{\text {sig }}$ is the predicted cross section for signal. A maximum likelihood fit is performed for the signal strength, and is shown in figure 8. Inclusion of the associated production of a single top quark with a Higgs boson in this study provides a $\approx 20 \%$ relative improvement in the expected upper limit on $\mathcal{B}(\mathrm{t} \rightarrow \mathrm{uH})$ with respect to the results obtained in an analysis of only t $\bar{t}$ events with top quark FCNC decays. 


\section{Summary}

A search for flavor-changing neutral currents in events with a top quark and the Higgs boson, corresponding to a data sample of $35.9 \mathrm{fb}^{-1}$ collected in proton-proton collisions at $\sqrt{s}=13 \mathrm{TeV}$, is presented. This is the first search to probe tH flavor-changing neutral current couplings in both associated production of a top quark with the Higgs boson and in top quark decays. Observed (expected) upper limits at $95 \%$ confidence level are set on the branching fractions of top quark decays, $\mathcal{B}(\mathrm{t} \rightarrow \mathrm{uH})<0.47 \%(0.34 \%)$ and $\mathcal{B}(\mathrm{t} \rightarrow \mathrm{cH})<0.47 \%(0.44 \%)$. These results provide a significant improvement over the previous limits set by CMS in the $\mathrm{H} \rightarrow \mathrm{b} \bar{b}$ channel, as well as represent the best limits for $\mathcal{B}(\mathrm{t} \rightarrow \mathrm{uH})$ at $\mathrm{CMS}$.

\section{Acknowledgments}

We congratulate our colleagues in the CERN accelerator departments for the excellent performance of the LHC and thank the technical and administrative staffs at CERN and at other CMS institutes for their contributions to the success of the CMS effort. In addition, we gratefully acknowledge the computing centres and personnel of the Worldwide LHC Computing Grid for delivering so effectively the computing infrastructure essential to our analyses. Finally, we acknowledge the enduring support for the construction and operation of the LHC and the CMS detector provided by the following funding agencies: BMWFW and FWF (Austria); FNRS and FWO (Belgium); CNPq, CAPES, FAPERJ, and FAPESP (Brazil); MES (Bulgaria); CERN; CAS, MoST, and NSFC (China); COLCIENCIAS (Colombia); MSES and CSF (Croatia); RPF (Cyprus); SENESCYT (Ecuador); MoER, ERC IUT, and ERDF (Estonia); Academy of Finland, MEC, and HIP (Finland); CEA and CNRS/IN2P3 (France); BMBF, DFG, and HGF (Germany); GSRT (Greece); OTKA and NIH (Hungary); DAE and DST (India); IPM (Iran); SFI (Ireland); INFN (Italy); MSIP and NRF (Republic of Korea); LAS (Lithuania); MOE and UM (Malaysia); BUAP, CINVESTAV, CONACYT, LNS, SEP, and UASLP-FAI (Mexico); MBIE (New Zealand); PAEC (Pakistan); MSHE and NSC (Poland); FCT (Portugal); JINR (Dubna); MON, RosAtom, RAS, RFBR and RAEP (Russia); MESTD (Serbia); SEIDI, CPAN, PCTI and FEDER (Spain); Swiss Funding Agencies (Switzerland); MST (Taipei); ThEPCenter, IPST, STAR, and NSTDA (Thailand); TUBITAK and TAEK (Turkey); NASU and SFFR (Ukraine); STFC (United Kingdom); DOE and NSF (U.S.A.).

Individuals have received support from the Marie-Curie programme and the European Research Council and Horizon 2020 Grant, contract No. 675440 (European Union); the Leventis Foundation; the A. P. Sloan Foundation; the Alexander von Humboldt Foundation; the Belgian Federal Science Policy Office; the Fonds pour la Formation à la Recherche dans l'Industrie et dans l'Agriculture (FRIA-Belgium); the Agentschap voor Innovatie door Wetenschap en Technologie (IWT-Belgium); the Ministry of Education, Youth and Sports (MEYS) of the Czech Republic; the Council of Science and Industrial Research, India; the HOMING PLUS programme of the Foundation for Polish Science, cofinanced from European Union, Regional Development Fund, the Mobility Plus programme of the Min- 
istry of Science and Higher Education, the National Science Center (Poland), contracts Harmonia 2014/14/M/ST2/00428, Opus 2014/13/B/ST2/02543, 2014/15/B/ST2/03998, and 2015/19/B/ST2/02861, Sonata-bis 2012/07/E/ST2/01406; the National Priorities Research Program by Qatar National Research Fund; the Programa Severo Ochoa del Principado de Asturias; the Thalis and Aristeia programmes cofinanced by EU-ESF and the Greek NSRF; the Rachadapisek Sompot Fund for Postdoctoral Fellowship, Chulalongkorn University and the Chulalongkorn Academic into Its 2nd Century Project Advancement Project (Thailand); the Welch Foundation, contract C-1845; and the Weston Havens Foundation (U.S.A.).

Open Access. This article is distributed under the terms of the Creative Commons Attribution License (CC-BY 4.0), which permits any use, distribution and reproduction in any medium, provided the original author(s) and source are credited.

\section{References}

[1] ATLAS collaboration, Observation of a new particle in the search for the standard model Higgs boson with the ATLAS detector at the LHC, Phys. Lett. B 716 (2012) 1 [arXiv:1207.7214] [INSPIRE].

[2] CMS collaboration, Observation of a new boson at a mass of $125 \mathrm{GeV}$ with the CMS experiment at the LHC, Phys. Lett. B 716 (2012) 30 [arXiv:1207.7235] [INSPIRE].

[3] CMS collaboration, Observation of a new boson with mass near $125 \mathrm{GeV}$ in pp collisions at $\sqrt{s}=7$ and $8 \mathrm{TeV}$, JHEP 06 (2013) 081 [arXiv:1303.4571] [INSPIRE].

[4] ATLAS, CMS collaboration, Measurements of the Higgs boson production and decay rates and constraints on its couplings from a combined ATLAS and CMS analysis of the LHC pp collision data at $\sqrt{s}=7$ and $8 \mathrm{TeV}$, JHEP 08 (2016) 045 [arXiv:1606.02266] [INSPIRE].

[5] S.L. Glashow, J. Iliopoulos and L. Maiani, Weak Interactions with Lepton-Hadron Symmetry, Phys. Rev. D 2 (1970) 1285 [InSPIRE].

[6] G. Eilam, J.L. Hewett and A. Soni, Rare decays of the top quark in the standard and two Higgs doublet models, Phys. Rev. D 44 (1991) 1473 [Erratum ibid. D 59 (1999) 039901] [INSPIRE].

[7] B. Mele, S. Petrarca and A. Soddu, A New evaluation of the $t \rightarrow c H$ decay width in the standard model, Phys. Lett. B 435 (1998) 401 [hep-ph/9805498] [INSPIRE].

[8] J.A. Aguilar-Saavedra, Top flavor-changing neutral interactions: theoretical expectations and experimental detection, Acta Phys. Polon. B 35 (2004) 2695 [hep-ph/0409342] [INSPIRE].

[9] F. Larios, R. Martinez and M.A. Perez, New physics effects in the flavor-changing neutral couplings of the top quark, Int. J. Mod. Phys. A 21 (2006) 3473 [hep-ph/0605003] [InSPIRE].

[10] ATLAS collaboration, Search for flavour-changing neutral current top quark decays $t \rightarrow H q$ in pp collisions at $\sqrt{s}=8 \mathrm{TeV}$ with the ATLAS detector, JHEP 12 (2015) 061 [arXiv: 1509.06047] [INSPIRE].

[11] ATLAS collaboration, Search for top quark decays $t \rightarrow q H$, with $H \rightarrow \gamma \gamma$, in $\sqrt{s}=13 \mathrm{TeV}$ pp collisions using the ATLAS detector, JHEP 10 (2017) 129 [arXiv:1707.01404] [INSPIRE]. 
[12] CMS collaboration, Search for top quark decays via Higgs-boson-mediated flavor-changing neutral currents in pp collisions at $\sqrt{s}=8$ TeV, JHEP 02 (2017) 079 [arXiv:1610.04857] [INSPIRE].

[13] R.D. Ball et al., Parton distributions with LHC data, Nucl. Phys. B 867 (2013) 244 [arXiv: 1207.1303] [INSPIRE].

[14] CMS collaboration, CMS luminosity measurements for the 2016 data taking period, CMS-PAS-LUM-17-001 (2017).

[15] CMS collaboration, The CMS experiment at the CERN LHC, 2008 JINST 3 S08004 [INSPIRE].

[16] J. Alwall et al., The automated computation of tree-level and next-to-leading order differential cross sections and their matching to parton shower simulations, JHEP 07 (2014) 079 [arXiv: 1405.0301] [INSPIRE].

[17] M.L. Mangano, M. Moretti, F. Piccinini and M. Treccani, Matching matrix elements and shower evolution for top-quark production in hadronic collisions, JHEP 01 (2007) 013 [hep-ph/0611129] [INSPIRE].

[18] J. Alwall et al., Comparative study of various algorithms for the merging of parton showers and matrix elements in hadronic collisions, Eur. Phys. J. C 53 (2008) 473 [arXiv:0706.2569] [INSPIRE].

[19] A. Alloul et al., FeynRules 2.0 - A complete toolbox for tree-level phenomenology, Comput. Phys. Commun. 185 (2014) 2250 [arXiv:1310.1921] [InSPIRE].

[20] C. Degrande et al., UFO - The Universal FeynRules Output, Comput. Phys. Commun. 183 (2012) 1201 [arXiv: 1108.2040] [INSPIRE].

[21] P. Nason, A New method for combining NLO QCD with shower Monte Carlo algorithms, JHEP 11 (2004) 040 [hep-ph/0409146] [INSPIRE].

[22] S. Frixione, P. Nason and C. Oleari, Matching NLO QCD computations with Parton Shower simulations: the POWHEG method, JHEP 11 (2007) 070 [arXiv:0709.2092] [INSPIRE].

[23] S. Alioli, P. Nason, C. Oleari and E. Re, A general framework for implementing NLO calculations in shower Monte Carlo programs: the POWHEG BOX, JHEP 06 (2010) 043 [arXiv: 1002.2581] [INSPIRE].

[24] S. Alioli, S.-O. Moch and P. Uwer, Hadronic top-quark pair-production with one jet and parton showering, JHEP 01 (2012) 137 [arXiv:1110.5251] [INSPIRE].

[25] M. Czakon and A. Mitov, Top ++ : a program for the calculation of the top-pair cross-section at hadron colliders, Comput. Phys. Commun. 185 (2014) 2930 [arXiv:1112.5675] [INSPIRE].

[26] M. Aliev, et al., HATHOR: HAdronic Top and Heavy quarks crOss section calculatoR, Comput. Phys. Commun. 182 (2011) 1034 [arXiv: 1007.1327] [INSPIRE].

[27] P. Kant et al., HatHor for single top-quark production: Updated predictions and uncertainty estimates for single top-quark production in hadronic collisions, Comput. Phys. Commun. 191 (2015) 74 [arXiv: 1406.4403] [INSPIRE].

[28] N. Kidonakis, Two-loop soft anomalous dimensions for single top quark associated production with a $W^{-}$or $H^{-}$, Phys. Rev. D 82 (2010) 054018 [arXiv:1005.4451] [InSPIRE].

[29] L. Barze et al., Neutral current Drell-Yan with combined QCD and electroweak corrections in the POWHEG BOX, Eur. Phys. J. C 73 (2013) 2474 [arXiv:1302.4606] [InSPIRE]. 
[30] T. Sjöstrand, S. Mrenna and P.Z. Skands, A brief introduction to PYTHIA 8.1, Comput. Phys. Commun. 178 (2008) 852 [arXiv:0710.3820] [INSPIRE].

[31] CMS collaboration, Event generator tunes obtained from underlying event and multiparton scattering measurements, Eur. Phys. J. C 76 (2016) 155 [arXiv:1512.00815] [INSPIRE].

[32] CMS collaboration, Investigations of the impact of the parton shower tuning in PYTHIA 8 in the modelling of $t \bar{t}$ at $\sqrt{s}=8$ and $13 \mathrm{TeV}$, CMS-PAS-TOP-16-021 (2016).

[33] NNPDF collaboration, R.D. Ball et al., Parton distributions for the LHC Run II, JHEP 04 (2015) 040 [arXiv:1410.8849] [INSPIRE].

[34] GEANT4 collaboration, S. Agostinelli et al., GEANT4 - a simulation toolkit, Nucl. Instrum. Meth. A 506 (2003) 250 [INSPIRE].

[35] CMS collaboration, Particle-flow reconstruction and global event description with the CMS detector, 2017 JINST 12 P10003 [arXiv:1706.04965] [INSPIRE].

[36] M. Cacciari, G.P. Salam and G. Soyez, The anti- $k_{t}$ jet clustering algorithm, JHEP 04 (2008) 063 [arXiv:0802.1189] [INSPIRE].

[37] M. Cacciari, G.P. Salam and G. Soyez, FastJet user manual, Eur. Phys. J. C 72 (2012) 1896 [arXiv:1111.6097] [INSPIRE].

[38] CMS collaboration, Determination of jet energy calibration and transverse momentum resolution in CMS, 2011 JINST 6 P11002 [arXiv:1107.4277] [INSPIRE].

[39] CMS Collaboration, Identification of b quark jets at the CMS Experiment in the LHC Run 2, CMS-PAS-BTV-15-001 (2015).

[40] T. Hastie, R. Tibshirani and J. Friedman, The elements of statistical learning, Springer-Verlag New York (2009).

[41] A. Hocker et al., TMVA - Toolkit for Multivariate Data Analysis, PoS(ACAT) 040 [physics/0703039] [INSPIRE].

[42] J. Butterworth et al., PDF 4 LHC recommendations for LHC Run II, J. Phys. G 43 (2016) 023001 [arXiv: 1510.03865] [INSPIRE].

[43] A.D. Martin, W.J. Stirling, R.S. Thorne and G. Watt, Uncertainties on $\alpha_{s}$ in global PDF analyses and implications for predicted hadronic cross sections, Eur. Phys. J. C 64 (2009) 653 [arXiv: 0905.3531] [INSPIRE].

[44] J. Gao et al., CT10 next-to-next-to-leading order global analysis of QCD, Phys. Rev. D 89 (2014) 033009 [arXiv:1302.6246] [INSPIRE].

[45] J.R. Christiansen and P.Z. Skands, String formation beyond leading colour, JHEP 08 (2015) 003 [arXiv: 1505.01681] [INSPIRE].

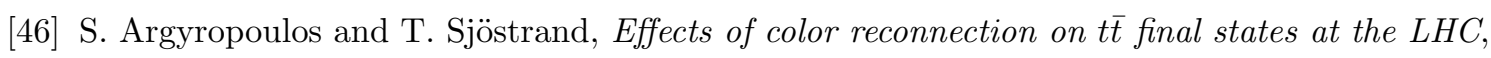
JHEP 11 (2014) 043 [arXiv: 1407.6653] [INSPIRE].

[47] CMS collaboration, Jet energy scale and resolution in the CMS experiment in pp collisions at $8 \mathrm{TeV}, 2017$ JINST $12 \mathrm{P} 02014$ [arXiv: 1607.03663] [INSPIRE].

[48] CMS collaboration, Measurement of differential cross sections for top quark pair production using the lepton+jets final state in proton-proton collisions at $13 \mathrm{TeV}$, Phys. Rev. D 95 (2017) 092001 [arXiv:1610.04191] [INSPIRE]. 
[49] A. Bredenstein, A. Denner, S. Dittmaier and S. Pozzorini, NLO QCD corrections to $p p \rightarrow t \bar{t} b \bar{b}+X$ at the LHC, Phys. Rev. Lett. 103 (2009) 012002 [arXiv:0905.0110] [InSPIRE].

[50] CMS collaboration, Measurements of $t \bar{t}$ cross sections in association with $b$ jets and inclusive jets and their ratio using dilepton final states in pp collisions at $\sqrt{s}=13 \mathrm{TeV}$, Phys. Lett. B 776 (2018) 355 [arXiv: 1705.10141] [INSPIRE].

[51] A.L. Read, Presentation of search results: the CL(s) technique, J. Phys. G 28 (2002) 2693 [INSPIRE].

[52] T. Junk, Confidence level computation for combining searches with small statistics, Nucl. Instrum. Meth. A 434 (1999) 435 [hep-ex/9902006] [INSPIRE].

[53] G. Cowan, K. Cranmer, E. Gross and O. Vitells, Asymptotic formulae for likelihood-based tests of new physics, Eur. Phys. J. C 71 (2011) 1554 [Erratum ibid. C 73 (2013) 2501] [arXiv: 1007.1727] [INSPIRE].

[54] J. Gao, C.S. Li and H.X. Zhu, Top quark decay at next-to-next-to leading order in QCD, Phys. Rev. Lett. 110 (2013) 042001 [arXiv: 1210.2808] [INSPIRE]. 


\section{The CMS collaboration}

\section{Yerevan Physics Institute, Yerevan, Armenia}

A.M. Sirunyan, A. Tumasyan

\section{Institut für Hochenergiephysik, Wien, Austria}

W. Adam, F. Ambrogi, E. Asilar, T. Bergauer, J. Brandstetter, E. Brondolin, M. Dragicevic, J. Erö, A. Escalante Del Valle, M. Flechl, M. Friedl, R. Frühwirth ${ }^{1}$, V.M. Ghete, J. Grossmann, J. Hrubec, M. Jeitler ${ }^{1}$, A. König, N. Krammer, I. Krätschmer, D. Liko, T. Madlener, I. Mikulec, E. Pree, N. Rad, H. Rohringer, J. Schieck ${ }^{1}$, R. Schöfbeck, M. Spanring, D. Spitzbart, A. Taurok, W. Waltenberger, J. Wittmann, C.-E. Wulz ${ }^{1}$, M. Zarucki

\section{Institute for Nuclear Problems, Minsk, Belarus}

V. Chekhovsky, V. Mossolov, J. Suarez Gonzalez

\section{Universiteit Antwerpen, Antwerpen, Belgium}

E.A. De Wolf, D. Di Croce, X. Janssen, J. Lauwers, M. Van De Klundert, H. Van Haevermaet, P. Van Mechelen, N. Van Remortel

\section{Vrije Universiteit Brussel, Brussel, Belgium}

S. Abu Zeid, F. Blekman, J. D'Hondt, I. De Bruyn, J. De Clercq, K. Deroover, G. Flouris, D. Lontkovskyi, S. Lowette, I. Marchesini, S. Moortgat, L. Moreels, Q. Python, K. Skovpen, S. Tavernier, W. Van Doninck, P. Van Mulders, I. Van Parijs

\section{Université Libre de Bruxelles, Bruxelles, Belgium}

D. Beghin, B. Bilin, H. Brun, B. Clerbaux, G. De Lentdecker, H. Delannoy, B. Dorney, G. Fasanella, L. Favart, R. Goldouzian, A. Grebenyuk, A.K. Kalsi, T. Lenzi, J. Luetic, T. Maerschalk, A. Marinov, T. Seva, E. Starling, C. Vander Velde, P. Vanlaer, D. Vannerom, R. Yonamine, F. Zenoni

\section{Ghent University, Ghent, Belgium}

T. Cornelis, D. Dobur, A. Fagot, M. Gul, I. Khvastunov², D. Poyraz, C. Roskas, S. Salva, D. Trocino, M. Tytgat, W. Verbeke, M. Vit, N. Zaganidis

\section{Université Catholique de Louvain, Louvain-la-Neuve, Belgium}

H. Bakhshiansohi, O. Bondu, S. Brochet, G. Bruno, C. Caputo, A. Caudron, P. David, S. De Visscher, C. Delaere, M. Delcourt, B. Francois, A. Giammanco, M. Komm, G. Krintiras, V. Lemaitre, A. Magitteri, A. Mertens, M. Musich, K. Piotrzkowski, L. Quertenmont, A. Saggio, M. Vidal Marono, S. Wertz, J. Zobec

\section{Centro Brasileiro de Pesquisas Fisicas, Rio de Janeiro, Brazil}

W.L. Aldá Júnior, F.L. Alves, G.A. Alves, L. Brito, G. Correia Silva, C. Hensel, A. Moraes, M.E. Pol, P. Rebello Teles

\section{Universidade do Estado do Rio de Janeiro, Rio de Janeiro, Brazil}

E. Belchior Batista Das Chagas, W. Carvalho, J. Chinellato ${ }^{3}$, E. Coelho, E.M. Da Costa, G.G. Da Silveira ${ }^{4}$, D. De Jesus Damiao, S. Fonseca De Souza, L.M. Huertas 
Guativa, H. Malbouisson, M. Melo De Almeida, C. Mora Herrera, L. Mundim, H. Nogima, L.J. Sanchez Rosas, A. Santoro, A. Sznajder, M. Thiel, E.J. Tonelli Manganote ${ }^{3}$, F. Torres Da Silva De Araujo, A. Vilela Pereira

Universidade Estadual Paulista ${ }^{a}$, Universidade Federal do $\mathrm{ABC}^{b}$, São Paulo, Brazil

S. Ahuja $^{a}$, C.A. Bernardes ${ }^{a}$, T.R. Fernandez Perez Tomei ${ }^{a}$, E.M. Gregores ${ }^{b}$, P.G. Mercadante ${ }^{b}$, S.F. Novaes ${ }^{a}$, Sandra S. Padula ${ }^{a}$, D. Romero Abad ${ }^{b}$, J.C. Ruiz Vargas ${ }^{a}$

Institute for Nuclear Research and Nuclear Energy, Bulgarian Academy of Sciences, Sofia, Bulgaria

A. Aleksandrov, R. Hadjiiska, P. Iaydjiev, M. Misheva, M. Rodozov, M. Shopova, G. Sultanov

University of Sofia, Sofia, Bulgaria

A. Dimitrov, L. Litov, B. Pavlov, P. Petkov

Beihang University, Beijing, China

W. Fang ${ }^{5}$, X. Gao ${ }^{5}$, L. Yuan

Institute of High Energy Physics, Beijing, China

M. Ahmad, J.G. Bian, G.M. Chen, H.S. Chen, M. Chen, Y. Chen, C.H. Jiang, D. Leggat, H. Liao, Z. Liu, F. Romeo, S.M. Shaheen, A. Spiezia, J. Tao, C. Wang, Z. Wang, E. Yazgan, H. Zhang, J. Zhao

State Key Laboratory of Nuclear Physics and Technology, Peking University, Beijing, China

Y. Ban, G. Chen, J. Li, Q. Li, S. Liu, Y. Mao, S.J. Qian, D. Wang, Z. Xu, F. Zhang ${ }^{5}$

Tsinghua University, Beijing, China

Y. Wang

Universidad de Los Andes, Bogota, Colombia

C. Avila, A. Cabrera, C.A. Carrillo Montoya, L.F. Chaparro Sierra, C. Florez, C.F. González Hernández, J.D. Ruiz Alvarez, M.A. Segura Delgado

University of Split, Faculty of Electrical Engineering, Mechanical Engineering and Naval Architecture, Split, Croatia

B. Courbon, N. Godinovic, D. Lelas, I. Puljak, P.M. Ribeiro Cipriano, T. Sculac

University of Split, Faculty of Science, Split, Croatia

Z. Antunovic, M. Kovac

Institute Rudjer Boskovic, Zagreb, Croatia

V. Brigljevic, D. Ferencek, K. Kadija, B. Mesic, A. Starodumov ${ }^{6}$, T. Susa

University of Cyprus, Nicosia, Cyprus

M.W. Ather, A. Attikis, G. Mavromanolakis, J. Mousa, C. Nicolaou, F. Ptochos, P.A. Razis, H. Rykaczewski 
Charles University, Prague, Czech Republic

M. Finger ${ }^{7}$, M. Finger Jr. ${ }^{7}$

Universidad San Francisco de Quito, Quito, Ecuador

E. Carrera Jarrin

Academy of Scientific Research and Technology of the Arab Republic of Egypt, Egyptian Network of High Energy Physics, Cairo, Egypt

A.A. Abdelalim ${ }^{8,9}$, Y. Mohammed ${ }^{10}$, E. Salama ${ }^{11,12}$

National Institute of Chemical Physics and Biophysics, Tallinn, Estonia

S. Bhowmik, R.K. Dewanjee, M. Kadastik, L. Perrini, M. Raidal, C. Veelken

Department of Physics, University of Helsinki, Helsinki, Finland

P. Eerola, H. Kirschenmann, J. Pekkanen, M. Voutilainen

Helsinki Institute of Physics, Helsinki, Finland

J. Havukainen, J.K. Heikkilä, T. Järvinen, V. Karimäki, R. Kinnunen, T. Lampén,

K. Lassila-Perini, S. Laurila, S. Lehti, T. Lindén, P. Luukka, T. Mäenpää, H. Siikonen,

E. Tuominen, J. Tuominiemi

Lappeenranta University of Technology, Lappeenranta, Finland

T. Tuuva

IRFU, CEA, Université Paris-Saclay, Gif-sur-Yvette, France

M. Besancon, F. Couderc, M. Dejardin, D. Denegri, J.L. Faure, F. Ferri, S. Ganjour,

S. Ghosh, A. Givernaud, P. Gras, G. Hamel de Monchenault, P. Jarry, C. Leloup, E. Locci,

M. Machet, J. Malcles, G. Negro, J. Rander, A. Rosowsky, M.Ö. Sahin, M. Titov

Laboratoire Leprince-Ringuet, Ecole polytechnique, CNRS/IN2P3, Université Paris-Saclay, Palaiseau, France

A. Abdulsalam ${ }^{13}$, C. Amendola, I. Antropov, S. Baffioni, F. Beaudette, P. Busson,

L. Cadamuro, C. Charlot, R. Granier de Cassagnac, M. Jo, I. Kucher, S. Lisniak,

A. Lobanov, J. Martin Blanco, M. Nguyen, C. Ochando, G. Ortona, P. Paganini, P. Pigard,

R. Salerno, J.B. Sauvan, Y. Sirois, A.G. Stahl Leiton, T. Strebler, Y. Yilmaz, A. Zabi,

A. Zghiche

Université de Strasbourg, CNRS, IPHC UMR 7178, F-67000 Strasbourg, France

J.-L. Agram ${ }^{14}$, J. Andrea, D. Bloch, J.-M. Brom, M. Buttignol, E.C. Chabert, C. Collard, E. Conte ${ }^{14}$, X. Coubez, F. Drouhin ${ }^{14}$, J.-C. Fontaine ${ }^{14}$, D. Gelé, U. Goerlach, M. Jansová, P. Juillot, A.-C. Le Bihan, N. Tonon, P. Van Hove

Centre de Calcul de l'Institut National de Physique Nucleaire et de Physique des Particules, CNRS/IN2P3, Villeurbanne, France

S. Gadrat 
Université de Lyon, Université Claude Bernard Lyon 1, CNRS-IN2P3, Institut de Physique Nucléaire de Lyon, Villeurbanne, France

S. Beauceron, C. Bernet, G. Boudoul, N. Chanon, R. Chierici, D. Contardo, P. Depasse, H. El Mamouni, J. Fay, L. Finco, S. Gascon, M. Gouzevitch, G. Grenier, B. Ille, F. Lagarde, I.B. Laktineh, M. Lethuillier, L. Mirabito, A.L. Pequegnot, S. Perries, A. Popov ${ }^{15}$, V. Sordini, M. Vander Donckt, S. Viret, S. Zhang

Georgian Technical University, Tbilisi, Georgia

T. Toriashvili ${ }^{16}$

Tbilisi State University, Tbilisi, Georgia

Z. Tsamalaidze ${ }^{7}$

RWTH Aachen University, I. Physikalisches Institut, Aachen, Germany

C. Autermann, L. Feld, M.K. Kiesel, K. Klein, M. Lipinski, M. Preuten, C. Schomakers, J. Schulz, M. Teroerde, B. Wittmer, V. Zhukov ${ }^{15}$

RWTH Aachen University, III. Physikalisches Institut A, Aachen, Germany

A. Albert, D. Duchardt, M. Endres, M. Erdmann, S. Erdweg, T. Esch, R. Fischer, A. Güth,

T. Hebbeker, C. Heidemann, K. Hoepfner, S. Knutzen, M. Merschmeyer, A. Meyer,

P. Millet, S. Mukherjee, T. Pook, M. Radziej, H. Reithler, M. Rieger, F. Scheuch,

D. Teyssier, S. Thüer

RWTH Aachen University, III. Physikalisches Institut B, Aachen, Germany

G. Flügge, B. Kargoll, T. Kress, A. Künsken, T. Müller, A. Nehrkorn, A. Nowack, C. Pistone, O. Pooth, A. Stahl ${ }^{17}$

\section{Deutsches Elektronen-Synchrotron, Hamburg, Germany}

M. Aldaya Martin, T. Arndt, C. Asawatangtrakuldee, K. Beernaert, O. Behnke, U. Behrens, A. Bermúdez Martínez, A.A. Bin Anuar, K. Borras ${ }^{18}$, V. Botta, A. Campbell, P. Connor, C. Contreras-Campana, F. Costanza, C. Diez Pardos, G. Eckerlin, D. Eckstein, T. Eichhorn, E. Eren, E. Gallo ${ }^{19}$, J. Garay Garcia, A. Geiser, J.M. Grados Luyando,

A. Grohsjean, P. Gunnellini, M. Guthoff, A. Harb, J. Hauk, M. Hempel ${ }^{20}$, H. Jung, M. Kasemann, J. Keaveney, C. Kleinwort, I. Korol, D. Krücker, W. Lange, A. Lelek, T. Lenz, K. Lipka, W. Lohmann ${ }^{20}$, R. Mankel, I.-A. Melzer-Pellmann, A.B. Meyer, M. Missiroli, G. Mittag, J. Mnich, A. Mussgiller, E. Ntomari, D. Pitzl, A. Raspereza, M. Savitskyi, P. Saxena, R. Shevchenko, N. Stefaniuk, G.P. Van Onsem, R. Walsh, Y. Wen, K. Wichmann, C. Wissing, O. Zenaiev

\section{University of Hamburg, Hamburg, Germany}

R. Aggleton, S. Bein, V. Blobel, M. Centis Vignali, T. Dreyer, E. Garutti, D. Gonzalez, J. Haller, A. Hinzmann, M. Hoffmann, A. Karavdina, R. Klanner, R. Kogler, N. Kovalchuk, S. Kurz, D. Marconi, M. Meyer, M. Niedziela, D. Nowatschin, F. Pantaleo ${ }^{17}$, T. Peiffer, A. Perieanu, C. Scharf, P. Schleper, A. Schmidt, S. Schumann, J. Schwandt, J. Sonneveld, H. Stadie, G. Steinbrück, F.M. Stober, M. Stöver, H. Tholen, D. Troendle, E. Usai, A. Vanhoefer, B. Vormwald 
Institut für Experimentelle Kernphysik, Karlsruhe, Germany

M. Akbiyik, C. Barth, M. Baselga, S. Baur, E. Butz, R. Caspart, T. Chwalek, F. Colombo, W. De Boer, A. Dierlamm, N. Faltermann, B. Freund, R. Friese, M. Giffels, M.A. Harrendorf, F. Hartmann ${ }^{17}$, S.M. Heindl, U. Husemann, F. Kasse ${ }^{17}$, S. Kudella, H. Mildner, M.U. Mozer, Th. Müller, M. Plagge, G. Quast, K. Rabbertz, M. Schröder, I. Shvetsov, G. Sieber, H.J. Simonis, R. Ulrich, S. Wayand, M. Weber, T. Weiler, S. Williamson, C. Wöhrmann, R. Wolf

Institute of Nuclear and Particle Physics (INPP), NCSR Demokritos, Aghia Paraskevi, Greece

G. Anagnostou, G. Daskalakis, T. Geralis, A. Kyriakis, D. Loukas, I. Topsis-Giotis

National and Kapodistrian University of Athens, Athens, Greece

G. Karathanasis, S. Kesisoglou, A. Panagiotou, N. Saoulidou, E. Tziaferi

National Technical University of Athens, Athens, Greece

K. Kousouris

University of Ioánnina, Ioánnina, Greece

I. Evangelou, C. Foudas, P. Gianneios, P. Katsoulis, P. Kokkas, S. Mallios, N. Manthos,

I. Papadopoulos, E. Paradas, J. Strologas, F.A. Triantis, D. Tsitsonis

MTA-ELTE Lendület CMS Particle and Nuclear Physics Group, Eötvös Loránd University, Budapest, Hungary

M. Csanad, N. Filipovic, G. Pasztor, O. Surányi, G.I. Veres ${ }^{21}$

Wigner Research Centre for Physics, Budapest, Hungary

G. Bencze, C. Hajdu, D. Horvath ${ }^{22}$, Á. Hunyadi, F. Sikler, V. Veszpremi, G. Vesztergombi ${ }^{21}$

Institute of Nuclear Research ATOMKI, Debrecen, Hungary

N. Beni, S. Czellar, J. Karancsi ${ }^{23}$, A. Makovec, J. Molnar, Z. Szillasi

Institute of Physics, University of Debrecen, Debrecen, Hungary

M. Bartók ${ }^{21}$, P. Raics, Z.L. Trocsanyi, B. Ujvari

Indian Institute of Science (IISc), Bangalore, India

S. Choudhury, J.R. Komaragiri

National Institute of Science Education and Research, Bhubaneswar, India

S. Bahinipati ${ }^{24}$, P. Mal, K. Mandal, A. Nayak ${ }^{25}$, D.K. Sahoo ${ }^{24}$, N. Sahoo, S.K. Swain

Panjab University, Chandigarh, India

S. Bansal, S.B. Beri, V. Bhatnagar, R. Chawla, N. Dhingra, A. Kaur, M. Kaur, S. Kaur, R. Kumar, P. Kumari, A. Mehta, J.B. Singh, G. Walia

University of Delhi, Delhi, India

Ashok Kumar, Aashaq Shah, A. Bhardwaj, S. Chauhan, B.C. Choudhary, R.B. Garg, S. Keshri, A. Kumar, S. Malhotra, M. Naimuddin, K. Ranjan, R. Sharma 
Saha Institute of Nuclear Physics, HBNI, Kolkata, India

R. Bhardwaj ${ }^{26}$, R. Bhattacharya, S. Bhattacharya, U. Bhawandeep ${ }^{26}$, D. Bhowmik, S. Dey, S. Dutt ${ }^{26}$, S. Dutta, S. Ghosh, N. Majumdar, A. Modak, K. Mondal, S. Mukhopadhyay, S. Nandan, A. Purohit, P.K. Rout, A. Roy, S. Roy Chowdhury, S. Sarkar, M. Sharan, B. Singh, S. Thakur ${ }^{26}$

\section{Indian Institute of Technology Madras, Madras, India}

P.K. Behera

Bhabha Atomic Research Centre, Mumbai, India

R. Chudasama, D. Dutta, V. Jha, V. Kumar, A.K. Mohanty ${ }^{17}$, P.K. Netrakanti, L.M. Pant, P. Shukla, A. Topkar

Tata Institute of Fundamental Research-A, Mumbai, India

T. Aziz, S. Dugad, B. Mahakud, S. Mitra, G.B. Mohanty, N. Sur, B. Sutar

Tata Institute of Fundamental Research-B, Mumbai, India

S. Banerjee, S. Bhattacharya, S. Chatterjee, P. Das, M. Guchait, Sa. Jain, S. Kumar, M. Maity ${ }^{27}$, G. Majumder, K. Mazumdar, T. Sarkar ${ }^{27}$, N. Wickramage ${ }^{28}$

Indian Institute of Science Education and Research (IISER), Pune, India

S. Chauhan, S. Dube, V. Hegde, A. Kapoor, K. Kothekar, S. Pandey, A. Rane, S. Sharma

Institute for Research in Fundamental Sciences (IPM), Tehran, Iran

S. Chenarani ${ }^{29}$, E. Eskandari Tadavani, S.M. Etesami ${ }^{29}$, M. Khakzad, M. Mohammadi Najafabadi, M. Naseri, S. Paktinat Mehdiabadi ${ }^{30}$, F. Rezaei Hosseinabadi, B. Safarzadeh ${ }^{31}$, M. Zeinali

University College Dublin, Dublin, Ireland

M. Felcini, M. Grunewald

INFN Sezione di Bari ${ }^{a}$, Università di Bari ${ }^{b}$, Politecnico di Bari ${ }^{c}$, Bari, Italy

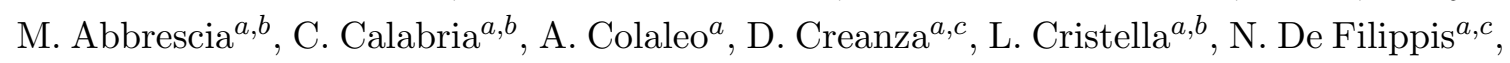
M. De Palma ${ }^{a, b}$, F. Errico ${ }^{a, b}$, L. Fiore ${ }^{a}$, G. Iaselli ${ }^{a, c}$, S. Lezki ${ }^{a, b}$, G. Maggi ${ }^{a, c}$, M. Maggi $^{a}$, B. Marangelli ${ }^{a, b}$, G. Miniello ${ }^{a, b}$, S. My ${ }^{a, b}$, S. Nuzzo ${ }^{a, b}$, A. Pompili ${ }^{a, b}$, G. Pugliese ${ }^{a, c}$, R. Radogna ${ }^{a}$, A. Ranieri ${ }^{a}$, G. Selvaggi ${ }^{a, b}$, A. Sharma ${ }^{a}$, L. Silvestris ${ }^{a, 17}$, R. $\operatorname{Venditti~}^{a}$, P. Verwilligen ${ }^{a}$, G. Zito $^{a}$

INFN Sezione di Bologna ${ }^{a}$, Università di Bologna ${ }^{b}$, Bologna, Italy

G. Abbiendi ${ }^{a}$, C. Battilana ${ }^{a, b}$, D. Bonacorsi ${ }^{a, b}$, L. Borgonovi $^{a, b}$, S. Braibant-Giacomellia $^{a, b}$, R. Campanini ${ }^{a, b}$, P. Capiluppi ${ }^{a, b}$, A. Castro ${ }^{a, b}$, F.R. Cavallo ${ }^{a}$, S.S. Chhibra ${ }^{a, b}$,

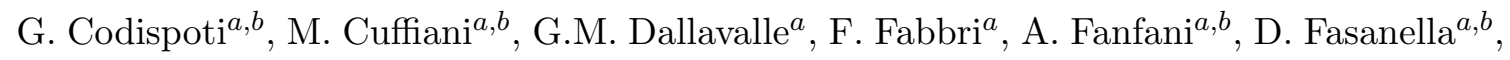
P. Giacomelli ${ }^{a}$, C. Grandi ${ }^{a}$, L. Guiducci ${ }^{a}, b$, F. Iemmi, S. Marcellini ${ }^{a}$, G. Masetti ${ }^{a}$,

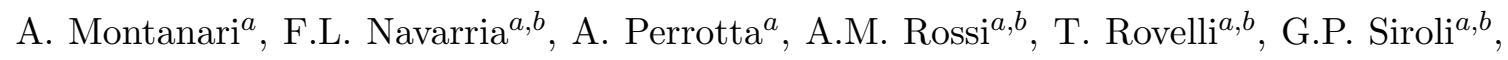
N. Tosi ${ }^{a}$

INFN Sezione di Catania ${ }^{a}$, Università di Catania ${ }^{b}$, Catania, Italy

S. Albergo ${ }^{a, b}$, S. Costa ${ }^{a, b}$, A. Di Mattia ${ }^{a}$, F. Giordano ${ }^{a, b}$, R. Potenza ${ }^{a, b}$, A. Tricomi ${ }^{a, b}$, C. Tuve $\mathrm{e}^{a, b}$ 
INFN Sezione di Firenze ${ }^{a}$, Università di Firenze ${ }^{b}$, Firenze, Italy

G. Barbagli ${ }^{a}$, K. Chatterjee ${ }^{a, b}$, V. Ciulli ${ }^{a, b}$, C. Civinini ${ }^{a}$, R. D’Alessandro ${ }^{a, b}$, E. Focardi $^{a, b}$, P. Lenzi ${ }^{a}, b$, M. Meschini ${ }^{a}$, S. Paoletti ${ }^{a}$, L. Russo $^{a, 32}$, G. Sguazzoni ${ }^{a}$, D. Strom ${ }^{a}$, L. Viliani $^{a}$

INFN Laboratori Nazionali di Frascati, Frascati, Italy

L. Benussi, S. Bianco, F. Fabbri, D. Piccolo, F. Primavera ${ }^{17}$

INFN Sezione di Genova ${ }^{a}$, Università di Genova ${ }^{b}$, Genova, Italy

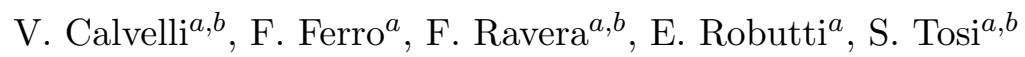

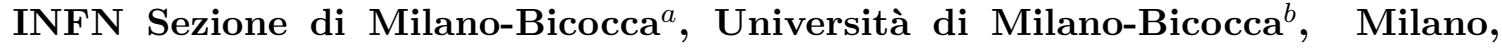
Italy

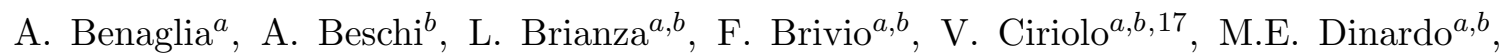
S. Fiorendi ${ }^{a, b}$, S. Gennai ${ }^{a}$, A. Ghezzi ${ }^{a, b}$, P. Govoni ${ }^{a, b}$, M. Malberti ${ }^{a, b}$, S. Malvezzi ${ }^{a}$, R.A. Manzoni ${ }^{a, b}$, D. Menasce ${ }^{a}$, L. Moroni ${ }^{a}$, M. Paganoni ${ }^{a, b}$, K. Pauwels ${ }^{a, b}$, D. Pedrini ${ }^{a}$, S. Pigazzini ${ }^{a, b, 33}$, S. Ragazzi ${ }^{a, b}$, T. Tabarelli de Fatis ${ }^{a, b}$

INFN Sezione di Napoli ${ }^{a}$, Università di Napoli 'Federico II' ${ }^{b}$, Napoli, Italy, Università della Basilicata ${ }^{c}$, Potenza, Italy, Università G. Marconi ${ }^{d}$, Roma, Italy

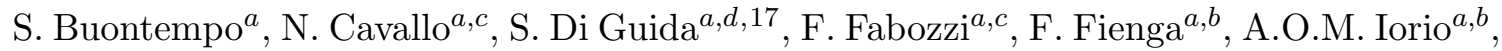

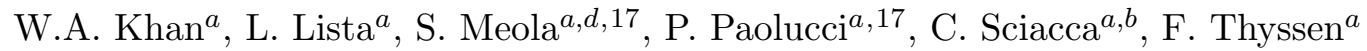

INFN Sezione di Padova ${ }^{a}$, Università di Padova ${ }^{b}$, Padova, Italy, Università di Trento $^{c}$, Trento, Italy

P. Azzi ${ }^{a}$, N. Bacchetta ${ }^{a}$, L. Benato ${ }^{a, b}$, M. Benettoni ${ }^{a}$, A. Boletti ${ }^{a, b}$, R. $\operatorname{Carlin}^{a, b}$, P. Checchia ${ }^{a}$, M. Dall'Osso ${ }^{a, b}$, P. De Castro Manzano ${ }^{a}$, T. Dorigo ${ }^{a}$, U. Dosselli ${ }^{a}$, F. Gasparini ${ }^{a, b}$, U. Gasparini ${ }^{a, b}$, A. Gozzelino ${ }^{a}$, S. Lacaprara ${ }^{a}$, P. Lujan, M. Margoni ${ }^{a, b}$, A.T. Meneguzzo ${ }^{a, b}$, N. Pozzobon ${ }^{a, b}$, P. Ronchese ${ }^{a, b}$, R. Rossin ${ }^{a, b}$, F. Simonetto ${ }^{a, b}$, A. Tiko, E. Torassa $^{a}$, M. Zanetti ${ }^{a, b}$, P. Zotto ${ }^{a, b}$, G. Zumerle ${ }^{a, b}$

INFN Sezione di Pavia ${ }^{a}$, Università di Pavia ${ }^{b}$, Pavia, Italy

A. Braghieri ${ }^{a}$, A. Magnani ${ }^{a}$, P. Montagna ${ }^{a, b}$, S.P. Ratti ${ }^{a, b}$, V. $\operatorname{Re}^{a}$, M. Ressegotti ${ }^{a, b}$, C. Riccardi ${ }^{a, b}$, P. Salvini ${ }^{a}$, I. Vai $^{a, b}$, P. Vitulo ${ }^{a, b}$

INFN Sezione di Perugia ${ }^{a}$, Università di Perugia ${ }^{b}$, Perugia, Italy

L. Alunni Solestizi ${ }^{a, b}$, M. Biasini ${ }^{a, b}$, G.M. Bilei ${ }^{a}$, C. Cecchi $^{a, b}$, D. Ciangottini ${ }^{a, b}$, L. Fanò $^{a, b}$, P. Lariccia ${ }^{a, b}$, R. Leonardi ${ }^{a, b}$, E. Manoni ${ }^{a}$, G. Mantovani ${ }^{a, b}$, V. Mariani ${ }^{a}, b$, M. Menichelli ${ }^{a}$, A. Rossi ${ }^{a, b}$, A. Santocchia ${ }^{a, b}$, D. Spiga ${ }^{a}$

INFN Sezione di Pisa ${ }^{a}$, Università di $\mathrm{Pisa}^{b}$, Scuola Normale Superiore di Pisa ${ }^{c}$, Pisa, Italy

K. Androsov ${ }^{a}$, P. Azzurri ${ }^{a, 17}$, G. Bagliesi ${ }^{a}$, L. Bianchini ${ }^{a}$, T. Boccali ${ }^{a}$, L. Borrello, R. Castaldi ${ }^{a}$, M.A. Ciocci ${ }^{a, b}$, R. Dell'Orso ${ }^{a}$, G. Fedi ${ }^{a}$, L. Giannini ${ }^{a, c}$, A. Giassi ${ }^{a}$, M.T. Grippo ${ }^{a, 32}$, F. Ligabue ${ }^{a, c}$, T. Lomtadze ${ }^{a}$, E. Manca $^{a, c}$, G. Mandorli $^{a, c}$, A. Messineo $^{a, b}$,

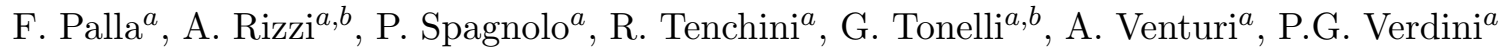




\section{INFN Sezione di Roma ${ }^{a}$, Sapienza Università di Roma ${ }^{b}$, Rome, Italy}

L. Barone $^{a, b}$, F. Cavallari ${ }^{a}$, M. Cipriani ${ }^{a, b}, \quad$ N. Daci ${ }^{a}$, D. Del Re Re $^{a, b}$ E. Di Marco $^{a, b}$, M. Diemoz ${ }^{a}$, S. Gelli ${ }^{a, b}$, E. Longo ${ }^{a, b}$, F. Margaroli ${ }^{a, b}$, B. Marzocchi ${ }^{a, b}$, P. Meridiani ${ }^{a}$, G. Organtini ${ }^{a, b}$, R. Paramatti ${ }^{a, b}$, F. Preiato ${ }^{a, b}$, S. Rahatlou ${ }^{a, b}$, C. Rovelli ${ }^{a}$, F. Santanastasio ${ }^{a, b}$

INFN Sezione di Torino ${ }^{a}$, Università di Torino ${ }^{b}$, Torino, Italy, Università del Piemonte Orientale ${ }^{c}$, Novara, Italy

N. Amapane ${ }^{a, b}$, R. Arcidiacono ${ }^{a, c}, \mathrm{~S}$. Argiro ${ }^{a, b}$, M. Arneodo ${ }^{a, c}$, N. Bartosik ${ }^{a}$, R. Bellan $^{a, b}$, C. Biino ${ }^{a}$, N. Cartiglia $^{a}$, F. Cenna ${ }^{a, b}$, M. Costa ${ }^{a, b}$, R. Covarellia ${ }^{a, b}$, A. Degano ${ }^{a, b}$, N. Demaria ${ }^{a}$, B. Kiani ${ }^{a, b}$, C. Mariotti ${ }^{a}$, S. Maselli ${ }^{a}$, E. Migliore ${ }^{a, b}$, V. Monaco ${ }^{a, b}$, E. Monteil ${ }^{a, b}$, M. Monteno ${ }^{a}$, M.M. Obertino ${ }^{a, b}$, L. Pacher $^{a}, b$, N. Pastrone ${ }^{a}$, M. Pelliccioni ${ }^{a}$, G.L. Pinna Angioni ${ }^{a, b}$, A. Romero ${ }^{a, b}$, M. Ruspa ${ }^{a, c}$, R. Sacchi ${ }^{a, b}$, K. Shchelina ${ }^{a, b}$, V. Sola ${ }^{a}$, A. Solano ${ }^{a, b}$, A. Staiano ${ }^{a}$, P. Traczyk ${ }^{a, b}$

INFN Sezione di Trieste ${ }^{a}$, Università di Trieste ${ }^{b}$, Trieste, Italy

S. Belforte ${ }^{a}$, M. Casarsa ${ }^{a}$, F. Cossutti ${ }^{a}$, G. Della Ricca ${ }^{a, b}$, A. Zanetti $^{a}$

Kyungpook National University, Daegu, Korea

D.H. Kim, G.N. Kim, M.S. Kim, J. Lee, S. Lee, S.W. Lee, C.S. Moon, Y.D. Oh, S. Sekmen, D.C. Son, Y.C. Yang

Chonnam National University, Institute for Universe and Elementary Particles, Kwangju, Korea

H. Kim, D.H. Moon, G. Oh

Hanyang University, Seoul, Korea

J.A. Brochero Cifuentes, J. Goh, T.J. Kim

Korea University, Seoul, Korea

S. Cho, S. Choi, Y. Go, D. Gyun, S. Ha, B. Hong, Y. Jo, Y. Kim, K. Lee, K.S. Lee, S. Lee, J. Lim, S.K. Park, Y. Roh

\section{Seoul National University, Seoul, Korea}

J. Almond, J. Kim, J.S. Kim, H. Lee, K. Lee, K. Nam, S.B. Oh, B.C. Radburn-Smith, S.h. Seo, U.K. Yang, H.D. Yoo, G.B. Yu

University of Seoul, Seoul, Korea

H. Kim, J.H. Kim, J.S.H. Lee, I.C. Park

Sungkyunkwan University, Suwon, Korea

Y. Choi, C. Hwang, J. Lee, I. Yu

Vilnius University, Vilnius, Lithuania

V. Dudenas, A. Juodagalvis, J. Vaitkus 
National Centre for Particle Physics, Universiti Malaya, Kuala Lumpur, Malaysia

I. Ahmed, Z.A. Ibrahim, M.A.B. Md $\mathrm{Ali}^{34}$, F. Mohamad Idris ${ }^{35}$, W.A.T. Wan Abdullah, M.N. Yusli, Z. Zolkapli

Centro de Investigacion y de Estudios Avanzados del IPN, Mexico City, Mexico Reyes-Almanza, R, Ramirez-Sanchez, G., Duran-Osuna, M. C., H. Castilla-Valdez, E. De La Cruz-Burelo, I. Heredia-De La Cruz ${ }^{36}$, Rabadan-Trejo, R. I., R. Lopez-Fernandez, J. Mejia Guisao, A. Sanchez-Hernandez

Universidad Iberoamericana, Mexico City, Mexico

S. Carrillo Moreno, C. Oropeza Barrera, F. Vazquez Valencia

Benemerita Universidad Autonoma de Puebla, Puebla, Mexico

J. Eysermans, I. Pedraza, H.A. Salazar Ibarguen, C. Uribe Estrada

Universidad Autónoma de San Luis Potosí, San Luis Potosí, Mexico

A. Morelos Pineda

University of Auckland, Auckland, New Zealand

D. Krofcheck

University of Canterbury, Christchurch, New Zealand

P.H. Butler

National Centre for Physics, Quaid-I-Azam University, Islamabad, Pakistan

A. Ahmad, M. Ahmad, Q. Hassan, H.R. Hoorani, A. Saddique, M.A. Shah, M. Shoaib, M. Waqas

National Centre for Nuclear Research, Swierk, Poland

H. Bialkowska, M. Bluj, B. Boimska, T. Frueboes, M. Górski, M. Kazana, K. Nawrocki, M. Szleper, P. Zalewski

Institute of Experimental Physics, Faculty of Physics, University of Warsaw, Warsaw, Poland

K. Bunkowski, A. Byszuk ${ }^{37}$, K. Doroba, A. Kalinowski, M. Konecki, J. Krolikowski, M. Misiura, M. Olszewski, A. Pyskir, M. Walczak

Laboratório de Instrumentação e Física Experimental de Partículas, Lisboa, Portugal

P. Bargassa, C. Beirão Da Cruz E Silva, A. Di Francesco, P. Faccioli, B. Galinhas, M. Gallinaro, J. Hollar, N. Leonardo, L. Lloret Iglesias, M.V. Nemallapudi, J. Seixas, G. Strong, O. Toldaiev, D. Vadruccio, J. Varela

Joint Institute for Nuclear Research, Dubna, Russia

S. Afanasiev, P. Bunin, M. Gavrilenko, I. Golutvin, I. Gorbunov, A. Kamenev, V. Karjavin, A. Lanev, A. Malakhov, V. Matveev ${ }^{38,39}$, P. Moisenz, V. Palichik, V. Perelygin, S. Shmatov, S. Shulha, N. Skatchkov, V. Smirnov, N. Voytishin, A. Zarubin 
Petersburg Nuclear Physics Institute, Gatchina (St. Petersburg), Russia

Y. Ivanov, V. Kim ${ }^{40}$, E. Kuznetsova ${ }^{41}$, P. Levchenko, V. Murzin, V. Oreshkin, I. Smirnov,

D. Sosnov, V. Sulimov, L. Uvarov, S. Vavilov, A. Vorobyev

Institute for Nuclear Research, Moscow, Russia

Yu. Andreev, A. Dermenev, S. Gninenko, N. Golubev, A. Karneyeu, M. Kirsanov,

N. Krasnikov, A. Pashenkov, D. Tlisov, A. Toropin

Institute for Theoretical and Experimental Physics, Moscow, Russia

V. Epshteyn, V. Gavrilov, N. Lychkovskaya, V. Popov, I. Pozdnyakov, G. Safronov, A. Spiridonov, A. Stepennov, V. Stolin, M. Toms, E. Vlasov, A. Zhokin

Moscow Institute of Physics and Technology, Moscow, Russia

T. Aushev, A. Bylinkin ${ }^{39}$

National Research Nuclear University 'Moscow Engineering Physics Institute' (MEPhI), Moscow, Russia

R. Chistov ${ }^{42}$, M. Danilov ${ }^{42}$, P. Parygin, D. Philippov, S. Polikarpov, E. Tarkovskii

P.N. Lebedev Physical Institute, Moscow, Russia

V. Andreev, M. Azarkin ${ }^{39}$, I. Dremin ${ }^{39}$, M. Kirakosyan ${ }^{39}$, S.V. Rusakov, A. Terkulov

Skobeltsyn Institute of Nuclear Physics, Lomonosov Moscow State University, Moscow, Russia

A. Baskakov, A. Belyaev, E. Boos, V. Bunichev, M. Dubinin ${ }^{43}$, L. Dudko, V. Klyukhin,

O. Kodolova, N. Korneeva, I. Lokhtin, I. Miagkov, S. Obraztsov, M. Perfilov, V. Savrin,

P. Volkov

Novosibirsk State University (NSU), Novosibirsk, Russia

V. Blinov ${ }^{44}$, D. Shtol ${ }^{44}$, Y. Skovpen ${ }^{44}$

State Research Center of Russian Federation, Institute for High Energy Physics of NRC \&quot;Kurchatov Institute\&quot;, Protvino, Russia

I. Azhgirey, I. Bayshev, S. Bitioukov, D. Elumakhov, A. Godizov, V. Kachanov, A. Kalinin,

D. Konstantinov, P. Mandrik, V. Petrov, R. Ryutin, A. Sobol, S. Troshin, N. Tyurin,

A. Uzunian, A. Volkov

National Research Tomsk Polytechnic University, Tomsk, Russia

A. Babaev

University of Belgrade, Faculty of Physics and Vinca Institute of Nuclear Sciences, Belgrade, Serbia

P. Adzic ${ }^{45}$, P. Cirkovic, D. Devetak, M. Dordevic, J. Milosevic

Centro de Investigaciones Energéticas Medioambientales y Tecnológicas (CIEMAT), Madrid, Spain

J. Alcaraz Maestre, I. Bachiller, M. Barrio Luna, M. Cerrada, N. Colino, B. De La Cruz, A. Delgado Peris, C. Fernandez Bedoya, J.P. Fernández Ramos, J. Flix, M.C. Fouz, O. Gonzalez Lopez, S. Goy Lopez, J.M. Hernandez, M.I. Josa, D. Moran, A. Pérez-Calero 
Yzquierdo, J. Puerta Pelayo, I. Redondo, L. Romero, M.S. Soares, A. Triossi, A. Álvarez Fernández

\section{Universidad Autónoma de Madrid, Madrid, Spain}

C. Albajar, J.F. de Trocóniz

\section{Universidad de Oviedo, Oviedo, Spain}

J. Cuevas, C. Erice, J. Fernandez Menendez, I. Gonzalez Caballero, J.R. González Fernández, E. Palencia Cortezon, S. Sanchez Cruz, P. Vischia, J.M. Vizan Garcia

Instituto de Física de Cantabria (IFCA), CSIC-Universidad de Cantabria, Santander, Spain

I.J. Cabrillo, A. Calderon, B. Chazin Quero, J. Duarte Campderros, M. Fernandez, P.J. Fernández Manteca, J. Garcia-Ferrero, A. García Alonso, G. Gomez, A. Lopez Virto, J. Marco, C. Martinez Rivero, P. Martinez Ruiz del Arbol, F. Matorras, J. Piedra Gomez, C. Prieels, T. Rodrigo, A. Ruiz-Jimeno, L. Scodellaro, N. Trevisani, I. Vila, R. Vilar Cortabitarte

\section{CERN, European Organization for Nuclear Research, Geneva, Switzerland}

D. Abbaneo, B. Akgun, E. Auffray, P. Baillon, A.H. Ball, D. Barney, J. Bendavid, M. Bianco, A. Bocci, C. Botta, T. Camporesi, R. Castello, M. Cepeda, G. Cerminara, E. Chapon, Y. Chen, D. d'Enterria, A. Dabrowski, V. Daponte, A. David, M. De Gruttola, A. De Roeck, N. Deelen, M. Dobson, T. du Pree, M. Dünser, N. Dupont, A. Elliott-Peisert, P. Everaerts, F. Fallavollita, G. Franzoni, J. Fulcher, W. Funk, D. Gigi, A. Gilbert, K. Gill, F. Glege, D. Gulhan, J. Hegeman, V. Innocente, A. Jafari, P. Janot, O. Karacheban ${ }^{20}$, J. Kieseler, V. Knünz, A. Kornmayer, M.J. Kortelainen, M. Krammer ${ }^{1}$, C. Lange, P. Lecoq, C. Lourenço, M.T. Lucchini, L. Malgeri, M. Mannelli, A. Martelli, F. Meijers, J.A. Merlin, S. Mersi, E. Meschi, P. Milenovic ${ }^{46}$, F. Moortgat, M. Mulders, H. Neugebauer, J. Ngadiuba, S. Orfanelli, L. Orsini, L. Pape, E. Perez, M. Peruzzi, A. Petrilli, G. Petrucciani, A. Pfeiffer, M. Pierini, F.M. Pitters, D. Rabady, A. Racz, T. Reis, G. Rolandi ${ }^{47}$, M. Rovere, H. Sakulin, C. Schäfer, C. Schwick, M. Seidel, M. Selvaggi, A. Sharma, P. Silva, P. Sphicas ${ }^{48}$, A. Stakia, J. Steggemann, M. Stoye, M. Tosi, D. Treille, A. Tsirou, V. Veckalns ${ }^{49}$, M. Verweij, W.D. Zeuner

\section{Paul Scherrer Institut, Villigen, Switzerland}

W. Bertl ${ }^{\dagger}$, L. Caminada ${ }^{50}$, K. Deiters, W. Erdmann, R. Horisberger, Q. Ingram, H.C. Kaestli, D. Kotlinski, U. Langenegger, T. Rohe, S.A. Wiederkehr

ETH Zurich - Institute for Particle Physics and Astrophysics (IPA), Zurich, Switzerland

M. Backhaus, L. Bäni, P. Berger, B. Casal, G. Dissertori, M. Dittmar, M. Donegà, C. Dorfer, C. Grab, C. Heidegger, D. Hits, J. Hoss, G. Kasieczka, T. Klijnsma, W. Lustermann, B. Mangano, M. Marionneau, M.T. Meinhard, D. Meister, F. Micheli, P. Musella, F. NessiTedaldi, F. Pandolfi, J. Pata, F. Pauss, G. Perrin, L. Perrozzi, M. Quittnat, M. Reichmann, D.A. Sanz Becerra, M. Schönenberger, L. Shchutska, V.R. Tavolaro, K. Theofilatos, M.L. Vesterbacka Olsson, R. Wallny, D.H. Zhu 
Universität Zürich, Zurich, Switzerland

T.K. Aarrestad, C. Amsler ${ }^{51}$, M.F. Canelli, A. De Cosa, R. Del Burgo, S. Donato, C. Galloni, T. Hreus, B. Kilminster, D. Pinna, G. Rauco, P. Robmann, D. Salerno, K. Schweiger, C. Seitz, Y. Takahashi, A. Zucchetta

National Central University, Chung-Li, Taiwan

V. Candelise, Y.H. Chang, K.y. Cheng, T.H. Doan, Sh. Jain, R. Khurana, C.M. Kuo, W. Lin, A. Pozdnyakov, S.S. Yu

National Taiwan University (NTU), Taipei, Taiwan

Arun Kumar, P. Chang, Y. Chao, K.F. Chen, P.H. Chen, F. Fiori, W.-S. Hou, Y. Hsiung, Y.F. Liu, R.-S. Lu, E. Paganis, A. Psallidas, A. Steen, J.f. Tsai

Chulalongkorn University, Faculty of Science, Department of Physics, Bangkok, Thailand

B. Asavapibhop, K. Kovitanggoon, G. Singh, N. Srimanobhas

Çukurova University, Physics Department, Science and Art Faculty, Adana, Turkey

A. Bat, F. Boran, S. Cerci ${ }^{52}$, S. Damarseckin, Z.S. Demiroglu, C. Dozen, I. Dumanoglu,

S. Girgis, G. Gokbulut, Y. Guler, I. $\operatorname{Hos}^{53}$, E.E. Kangal ${ }^{54}$, O. Kara, A. Kayis Topaksu,

U. Kiminsu, M. Oglakci, G. Onengut, K. Ozdemir ${ }^{55}$, D. Sunar Cerci ${ }^{52}$, B. Tali ${ }^{52}$, U.G. Tok,

S. Turkcapar, I.S. Zorbakir, C. Zorbilmez

Middle East Technical University, Physics Department, Ankara, Turkey

G. Karapinar ${ }^{56}$, K. Ocalan ${ }^{57}$, M. Yalvac, M. Zeyrek

Bogazici University, Istanbul, Turkey

E. Gülmez, M. Kaya ${ }^{58}$, O. Kaya ${ }^{59}$, S. Tekten, E.A. Yetkin ${ }^{60}$

Istanbul Technical University, Istanbul, Turkey

M.N. Agaras, S. Atay, A. Cakir, K. Cankocak, Y. Komurcu

Institute for Scintillation Materials of National Academy of Science of Ukraine, Kharkov, Ukraine

B. Grynyov

National Scientific Center, Kharkov Institute of Physics and Technology, Kharkov, Ukraine

L. Levchuk

University of Bristol, Bristol, United Kingdom

F. Ball, L. Beck, J.J. Brooke, D. Burns, E. Clement, D. Cussans, O. Davignon, H. Flacher,

J. Goldstein, G.P. Heath, H.F. Heath, L. Kreczko, D.M. Newbold ${ }^{61}$, S. Paramesvaran,

T. Sakuma, S. Seif El Nasr-storey, D. Smith, V.J. Smith 


\section{Rutherford Appleton Laboratory, Didcot, United Kingdom}

K.W. Bell, A. Belyaev ${ }^{62}$, C. Brew, R.M. Brown, L. Calligaris, D. Cieri, D.J.A. Cockerill, J.A. Coughlan, K. Harder, S. Harper, J. Linacre, E. Olaiya, D. Petyt, C.H. ShepherdThemistocleous, A. Thea, I.R. Tomalin, T. Williams, W.J. Womersley

\section{Imperial College, London, United Kingdom}

G. Auzinger, R. Bainbridge, P. Bloch, J. Borg, S. Breeze, O. Buchmuller, A. Bundock, S. Casasso, M. Citron, D. Colling, L. Corpe, P. Dauncey, G. Davies, M. Della Negra, R. Di Maria, Y. Haddad, G. Hall, G. Iles, T. James, R. Lane, C. Laner, L. Lyons, A.-M. Magnan, S. Malik, L. Mastrolorenzo, T. Matsushita, J. Nash ${ }^{63}$, A. Nikitenko ${ }^{6}$, V. Palladino, M. Pesaresi, D.M. Raymond, A. Richards, A. Rose, E. Scott, C. Seez, A. Shtipliyski, S. Summers, A. Tapper, K. Uchida, M. Vazquez Acosta ${ }^{64}$, T. Virdee ${ }^{17}$, N. Wardle, D. Winterbottom, J. Wright, S.C. Zenz

\section{Brunel University, Uxbridge, United Kingdom}

J.E. Cole, P.R. Hobson, A. Khan, P. Kyberd, A. Morton, I.D. Reid, L. Teodorescu, S. Zahid Baylor University, Waco, U.S.A.

A. Borzou, K. Call, J. Dittmann, K. Hatakeyama, H. Liu, N. Pastika, C. Smith

Catholic University of America, Washington DC, U.S.A.

R. Bartek, A. Dominguez

The University of Alabama, Tuscaloosa, U.S.A.

A. Buccilli, S.I. Cooper, C. Henderson, P. Rumerio, C. West

Boston University, Boston, U.S.A.

D. Arcaro, A. Avetisyan, T. Bose, D. Gastler, D. Rankin, C. Richardson, J. Rohlf, L. Sulak, D. Zou

\section{Brown University, Providence, U.S.A.}

G. Benelli, D. Cutts, M. Hadley, J. Hakala, U. Heintz, J.M. Hogan, K.H.M. Kwok, E. Laird, G. Landsberg, J. Lee, Z. Mao, M. Narain, J. Pazzini, S. Piperov, S. Sagir, R. Syarif, D. Yu

University of California, Davis, Davis, U.S.A.

R. Band, C. Brainerd, R. Breedon, D. Burns, M. Calderon De La Barca Sanchez, M. Chertok, J. Conway, R. Conway, P.T. Cox, R. Erbacher, C. Flores, G. Funk, W. Ko, R. Lander, C. Mclean, M. Mulhearn, D. Pellett, J. Pilot, S. Shalhout, M. Shi, J. Smith, D. Stolp, D. Taylor, K. Tos, M. Tripathi, Z. Wang

University of California, Los Angeles, U.S.A.

M. Bachtis, C. Bravo, R. Cousins, A. Dasgupta, A. Florent, J. Hauser, M. Ignatenko, N. Mccoll, S. Regnard, D. Saltzberg, C. Schnaible, V. Valuev

University of California, Riverside, Riverside, U.S.A.

E. Bouvier, K. Burt, R. Clare, J. Ellison, J.W. Gary, S.M.A. Ghiasi Shirazi, G. Hanson, G. Karapostoli, E. Kennedy, F. Lacroix, O.R. Long, M. Olmedo Negrete, M.I. Paneva, W. Si, L. Wang, H. Wei, S. Wimpenny, B. R. Yates 
University of California, San Diego, La Jolla, U.S.A.

J.G. Branson, S. Cittolin, M. Derdzinski, R. Gerosa, D. Gilbert, B. Hashemi, A. Holzner, D. Klein, G. Kole, V. Krutelyov, J. Letts, M. Masciovecchio, D. Olivito, S. Padhi, M. Pieri, M. Sani, V. Sharma, S. Simon, M. Tadel, A. Vartak, S. Wasserbaech ${ }^{65}$, J. Wood, F. Würthwein, A. Yagil, G. Zevi Della Porta

University of California, Santa Barbara - Department of Physics, Santa Barbara, U.S.A.

N. Amin, R. Bhandari, J. Bradmiller-Feld, C. Campagnari, A. Dishaw, V. Dutta, M. Franco Sevilla, L. Gouskos, R. Heller, J. Incandela, A. Ovcharova, H. Qu, J. Richman, D. Stuart, I. Suarez, J. Yoo

California Institute of Technology, Pasadena, U.S.A.

D. Anderson, A. Bornheim, J. Bunn, I. Dutta, J.M. Lawhorn, H.B. Newman, T. Q. Nguyen, C. Pena, M. Spiropulu, J.R. Vlimant, R. Wilkinson, S. Xie, Z. Zhang, R.Y. Zhu

Carnegie Mellon University, Pittsburgh, U.S.A.

M.B. Andrews, T. Ferguson, T. Mudholkar, M. Paulini, J. Russ, M. Sun, H. Vogel, I. Vorobiev, M. Weinberg

University of Colorado Boulder, Boulder, U.S.A.

J.P. Cumalat, W.T. Ford, F. Jensen, A. Johnson, M. Krohn, S. Leontsinis, E. Macdonald, T. Mulholland, K. Stenson, S.R. Wagner

Cornell University, Ithaca, U.S.A.

J. Alexander, J. Chaves, Y. Cheng, J. Chu, S. Dittmer, K. Mcdermott, N. Mirman, J.R. Patterson, D. Quach, A. Rinkevicius, A. Ryd, L. Skinnari, L. Soffi, S.M. Tan, Z. Tao, J. Thom, J. Tucker, P. Wittich, M. Zientek

Fermi National Accelerator Laboratory, Batavia, U.S.A.

S. Abdullin, M. Albrow, M. Alyari, G. Apollinari, A. Apresyan, A. Apyan, S. Banerjee, L.A.T. Bauerdick, A. Beretvas, J. Berryhill, P.C. Bhat, G. Bolla ${ }^{\dagger}$, K. Burkett, J.N. Butler, A. Canepa, G.B. Cerati, H.W.K. Cheung, F. Chlebana, M. Cremonesi, J. Duarte, V.D. Elvira, J. Freeman, Z. Gecse, E. Gottschalk, L. Gray, D. Green, S. Grünendahl, O. Gutsche, J. Hanlon, R.M. Harris, S. Hasegawa, J. Hirschauer, Z. Hu, B. Jayatilaka, S. Jindariani, M. Johnson, U. Joshi, B. Klima, B. Kreis, S. Lammel, D. Lincoln, R. Lipton, M. Liu, T. Liu, R. Lopes De Sá, J. Lykken, K. Maeshima, N. Magini, J.M. Marraffino, D. Mason, P. McBride, P. Merkel, S. Mrenna, S. Nahn, V. O’Dell, K. Pedro, O. Prokofyev, G. Rakness, L. Ristori, A. Savoy-Navarro ${ }^{66}$, B. Schneider, E. Sexton-Kennedy, A. Soha, W.J. Spalding, L. Spiegel, S. Stoynev, J. Strait, N. Strobbe, L. Taylor, S. Tkaczyk, N.V. Tran, L. Uplegger, E.W. Vaandering, C. Vernieri, M. Verzocchi, R. Vidal, M. Wang, H.A. Weber, A. Whitbeck, W. Wu

University of Florida, Gainesville, U.S.A.

D. Acosta, P. Avery, P. Bortignon, D. Bourilkov, A. Brinkerhoff, A. Carnes, M. Carver, D. Curry, R.D. Field, I.K. Furic, S.V. Gleyzer, B.M. Joshi, J. Konigsberg, A. Korytov, 
K. Kotov, P. Ma, K. Matchev, H. Mei, G. Mitselmakher, K. Shi, D. Sperka, N. Terentyev, L. Thomas, J. Wang, S. Wang, J. Yelton

Florida International University, Miami, U.S.A.

Y.R. Joshi, S. Linn, P. Markowitz, J.L. Rodriguez

Florida State University, Tallahassee, U.S.A.

A. Ackert, T. Adams, A. Askew, S. Hagopian, V. Hagopian, K.F. Johnson, T. Kolberg, G. Martinez, T. Perry, H. Prosper, A. Saha, A. Santra, V. Sharma, R. Yohay

Florida Institute of Technology, Melbourne, U.S.A.

M.M. Baarmand, V. Bhopatkar, S. Colafranceschi, M. Hohlmann, D. Noonan, T. Roy, F. Yumiceva

University of Illinois at Chicago (UIC), Chicago, U.S.A.

M.R. Adams, L. Apanasevich, D. Berry, R.R. Betts, R. Cavanaugh, X. Chen, O. Evdokimov, C.E. Gerber, D.A. Hangal, D.J. Hofman, K. Jung, J. Kamin, I.D. Sandoval Gonzalez, M.B. Tonjes, H. Trauger, N. Varelas, H. Wang, Z. Wu, J. Zhang

The University of Iowa, Iowa City, U.S.A.

B. Bilki ${ }^{67}$, W. Clarida, K. Dilsiz ${ }^{68}$, S. Durgut, R.P. Gandrajula, M. Haytmyradov, V. Khristenko, J.-P. Merlo, H. Mermerkaya ${ }^{69}$, A. Mestvirishvili, A. Moeller, J. Nachtman, H. Ogul ${ }^{70}$, Y. Onel, F. Ozok ${ }^{71}$, A. Penzo, C. Snyder, E. Tiras, J. Wetzel, K. Yi

Johns Hopkins University, Baltimore, U.S.A.

B. Blumenfeld, A. Cocoros, N. Eminizer, D. Fehling, L. Feng, A.V. Gritsan, P. Maksimovic, J. Roskes, U. Sarica, M. Swartz, M. Xiao, C. You

The University of Kansas, Lawrence, U.S.A.

A. Al-bataineh, P. Baringer, A. Bean, S. Boren, J. Bowen, J. Castle, S. Khalil, A. Kropivnitskaya, D. Majumder, W. Mcbrayer, M. Murray, C. Rogan, C. Royon, S. Sanders, E. Schmitz, J.D. Tapia Takaki, Q. Wang

Kansas State University, Manhattan, U.S.A.

A. Ivanov, K. Kaadze, Y. Maravin, A. Mohammadi, L.K. Saini, N. Skhirtladze

Lawrence Livermore National Laboratory, Livermore, U.S.A.

F. Rebassoo, D. Wright

University of Maryland, College Park, U.S.A.

A. Baden, O. Baron, A. Belloni, S.C. Eno, Y. Feng, C. Ferraioli, N.J. Hadley, S. Jabeen, G.Y. Jeng, R.G. Kellogg, J. Kunkle, A.C. Mignerey, F. Ricci-Tam, Y.H. Shin, A. Skuja, S.C. Tonwar

\section{Massachusetts Institute of Technology, Cambridge, U.S.A.}

D. Abercrombie, B. Allen, V. Azzolini, R. Barbieri, A. Baty, G. Bauer, R. Bi, S. Brandt, W. Busza, I.A. Cali, M. D'Alfonso, Z. Demiragli, G. Gomez Ceballos, M. Goncharov, P. Harris, D. Hsu, M. Hu, Y. Iiyama, G.M. Innocenti, M. Klute, D. Kovalskyi, Y.-J. Lee, A. Levin, P.D. Luckey, B. Maier, A.C. Marini, C. Mcginn, C. Mironov, S. Narayanan, 
X. Niu, C. Paus, C. Roland, G. Roland, J. Salfeld-Nebgen, G.S.F. Stephans, K. Sumorok, K. Tatar, D. Velicanu, J. Wang, T.W. Wang, B. Wyslouch

University of Minnesota, Minneapolis, U.S.A.

A.C. Benvenuti, R.M. Chatterjee, A. Evans, P. Hansen, J. Hiltbrand, S. Kalafut, Y. Kubota, Z. Lesko, J. Mans, S. Nourbakhsh, N. Ruckstuhl, R. Rusack, J. Turkewitz, M.A. Wadud

University of Mississippi, Oxford, U.S.A.

J.G. Acosta, S. Oliveros

University of Nebraska-Lincoln, Lincoln, U.S.A.

E. Avdeeva, K. Bloom, D.R. Claes, C. Fangmeier, F. Golf, R. Gonzalez Suarez, R. Kamalieddin, I. Kravchenko, J. Monroy, J.E. Siado, G.R. Snow, B. Stieger

State University of New York at Buffalo, Buffalo, U.S.A.

J. Dolen, A. Godshalk, C. Harrington, I. Iashvili, D. Nguyen, A. Parker, S. Rappoccio,

B. Roozbahani

Northeastern University, Boston, U.S.A.

G. Alverson, E. Barberis, C. Freer, A. Hortiangtham, A. Massironi, D.M. Morse, T. Orimoto, R. Teixeira De Lima, T. Wamorkar, B. Wang, A. Wisecarver, D. Wood

Northwestern University, Evanston, U.S.A.

S. Bhattacharya, O. Charaf, K.A. Hahn, N. Mucia, N. Odell, M.H. Schmitt, K. Sung, M. Trovato, M. Velasco

University of Notre Dame, Notre Dame, U.S.A.

R. Bucci, N. Dev, M. Hildreth, K. Hurtado Anampa, C. Jessop, D.J. Karmgard, N. Kellams, K. Lannon, W. Li, N. Loukas, N. Marinelli, F. Meng, C. Mueller, Y. Musienko ${ }^{38}$, M. Planer, A. Reinsvold, R. Ruchti, P. Siddireddy, G. Smith, S. Taroni, M. Wayne, A. Wightman, M. Wolf, A. Woodard

The Ohio State University, Columbus, U.S.A.

J. Alimena, L. Antonelli, B. Bylsma, L.S. Durkin, S. Flowers, B. Francis, A. Hart, C. Hill, W. Ji, T.Y. Ling, B. Liu, W. Luo, B.L. Winer, H.W. Wulsin

Princeton University, Princeton, U.S.A.

S. Cooperstein, O. Driga, P. Elmer, J. Hardenbrook, P. Hebda, S. Higginbotham, A. Kalogeropoulos, D. Lange, J. Luo, D. Marlow, K. Mei, I. Ojalvo, J. Olsen, C. Palmer, P. Piroué, D. Stickland, C. Tully

University of Puerto Rico, Mayaguez, U.S.A.

S. Malik, S. Norberg

Purdue University, West Lafayette, U.S.A.

A. Barker, V.E. Barnes, S. Das, S. Folgueras, L. Gutay, M. Jones, A.W. Jung, A. Khatiwada, D.H. Miller, N. Neumeister, C.C. Peng, H. Qiu, J.F. Schulte, J. Sun, F. Wang, R. Xiao, W. Xie 
Purdue University Northwest, Hammond, U.S.A.

T. Cheng, N. Parashar, J. Stupak

Rice University, Houston, U.S.A.

Z. Chen, K.M. Ecklund, S. Freed, F.J.M. Geurts, M. Guilbaud, M. Kilpatrick, W. Li, B. Michlin, B.P. Padley, J. Roberts, J. Rorie, W. Shi, Z. Tu, J. Zabel, A. Zhang

University of Rochester, Rochester, U.S.A.

A. Bodek, P. de Barbaro, R. Demina, Y.t. Duh, T. Ferbel, M. Galanti, A. Garcia-Bellido, J. Han, O. Hindrichs, A. Khukhunaishvili, K.H. Lo, P. Tan, M. Verzetti

The Rockefeller University, New York, U.S.A.

R. Ciesielski, K. Goulianos, C. Mesropian

Rutgers, The State University of New Jersey, Piscataway, U.S.A.

A. Agapitos, J.P. Chou, Y. Gershtein, T.A. Gómez Espinosa, E. Halkiadakis, M. Heindl,

E. Hughes, S. Kaplan, R. Kunnawalkam Elayavalli, S. Kyriacou, A. Lath, R. Montalvo,

K. Nash, M. Osherson, H. Saka, S. Salur, S. Schnetzer, D. Sheffield, S. Somalwar, R. Stone,

S. Thomas, P. Thomassen, M. Walker

University of Tennessee, Knoxville, U.S.A.

A.G. Delannoy, J. Heideman, G. Riley, K. Rose, S. Spanier, K. Thapa

Texas A\&M University, College Station, U.S.A.

O. Bouhali ${ }^{72}$, A. Castaneda Hernandez ${ }^{72}$, A. Celik, M. Dalchenko, M. De Mattia, A. Delgado, S. Dildick, R. Eusebi, J. Gilmore, T. Huang, T. Kamon ${ }^{73}$, R. Mueller, Y. Pakhotin, R. Patel, A. Perloff, L. Perniè, D. Rathjens, A. Safonov, A. Tatarinov, K.A. Ulmer

Texas Tech University, Lubbock, U.S.A.

N. Akchurin, J. Damgov, F. De Guio, P.R. Dudero, J. Faulkner, E. Gurpinar, S. Kunori, K. Lamichhane, S.W. Lee, T. Mengke, S. Muthumuni, T. Peltola, S. Undleeb, I. Volobouev, Z. Wang

Vanderbilt University, Nashville, U.S.A.

S. Greene, A. Gurrola, R. Janjam, W. Johns, C. Maguire, A. Melo, H. Ni, K. Padeken, P. Sheldon, S. Tuo, J. Velkovska, Q. Xu

University of Virginia, Charlottesville, U.S.A.

M.W. Arenton, P. Barria, B. Cox, R. Hirosky, M. Joyce, A. Ledovskoy, H. Li, C. Neu, T. Sinthuprasith, Y. Wang, E. Wolfe, F. Xia

Wayne State University, Detroit, U.S.A.

R. Harr, P.E. Karchin, N. Poudyal, J. Sturdy, P. Thapa, S. Zaleski

University of Wisconsin - Madison, Madison, WI, U.S.A.

M. Brodski, J. Buchanan, C. Caillol, D. Carlsmith, S. Dasu, L. Dodd, S. Duric, B. Gomber,

M. Grothe, M. Herndon, A. Hervé, U. Hussain, P. Klabbers, A. Lanaro, A. Levine, K. Long,

R. Loveless, V. Rekovic, T. Ruggles, A. Savin, N. Smith, W.H. Smith, N. Woods 
$\dagger$ : Deceased

1: Also at Vienna University of Technology, Vienna, Austria

2: Also at IRFU, CEA, Université Paris-Saclay, Gif-sur-Yvette, France

3: Also at Universidade Estadual de Campinas, Campinas, Brazil

4: Also at Federal University of Rio Grande do Sul, Porto Alegre, Brazil

5: Also at Université Libre de Bruxelles, Bruxelles, Belgium

6: Also at Institute for Theoretical and Experimental Physics, Moscow, Russia

7: Also at Joint Institute for Nuclear Research, Dubna, Russia

8: Also at Helwan University, Cairo, Egypt

9: Now at Zewail City of Science and Technology, Zewail, Egypt

10: Now at Fayoum University, El-Fayoum, Egypt

11: Also at British University in Egypt, Cairo, Egypt

12: Now at Ain Shams University, Cairo, Egypt

13: Also at Department of Physics, King Abdulaziz University, Jeddah, Saudi Arabia

14: Also at Université de Haute Alsace, Mulhouse, France

15: Also at Skobeltsyn Institute of Nuclear Physics, Lomonosov Moscow State University, Moscow, Russia

16: Also at Tbilisi State University, Tbilisi, Georgia

17: Also at CERN, European Organization for Nuclear Research, Geneva, Switzerland

18: Also at RWTH Aachen University, III. Physikalisches Institut A, Aachen, Germany

19: Also at University of Hamburg, Hamburg, Germany

20: Also at Brandenburg University of Technology, Cottbus, Germany

21: Also at MTA-ELTE Lendület CMS Particle and Nuclear Physics Group, Eötvös Loránd University, Budapest, Hungary

22: Also at Institute of Nuclear Research ATOMKI, Debrecen, Hungary

23: Also at Institute of Physics, University of Debrecen, Debrecen, Hungary

24: Also at Indian Institute of Technology Bhubaneswar, Bhubaneswar, India

25: Also at Institute of Physics, Bhubaneswar, India

26: Also at Shoolini University, Solan, India

27: Also at University of Visva-Bharati, Santiniketan, India

28: Also at University of Ruhuna, Matara, Sri Lanka

29: Also at Isfahan University of Technology, Isfahan, Iran

30: Also at Yazd University, Yazd, Iran

31: Also at Plasma Physics Research Center, Science and Research Branch, Islamic Azad University, Tehran, Iran

32: Also at Università degli Studi di Siena, Siena, Italy

33: Also at INFN Sezione di Milano-Bicocca; Università di Milano-Bicocca, Milano, Italy

34: Also at International Islamic University of Malaysia, Kuala Lumpur, Malaysia

35: Also at Malaysian Nuclear Agency, MOSTI, Kajang, Malaysia

36: Also at Consejo Nacional de Ciencia y Tecnología, Mexico city, Mexico

37: Also at Warsaw University of Technology, Institute of Electronic Systems, Warsaw, Poland

38: Also at Institute for Nuclear Research, Moscow, Russia

39: Now at National Research Nuclear University 'Moscow Engineering Physics Institute' (MEPhI), Moscow, Russia

40: Also at St. Petersburg State Polytechnical University, St. Petersburg, Russia

41: Also at University of Florida, Gainesville, U.S.A.

42: Also at P.N. Lebedev Physical Institute, Moscow, Russia

43: Also at California Institute of Technology, Pasadena, U.S.A. 
44: Also at Budker Institute of Nuclear Physics, Novosibirsk, Russia

45: Also at Faculty of Physics, University of Belgrade, Belgrade, Serbia

46: Also at University of Belgrade, Faculty of Physics and Vinca Institute of Nuclear Sciences, Belgrade, Serbia

47: Also at Scuola Normale e Sezione dell'INFN, Pisa, Italy

48: Also at National and Kapodistrian University of Athens, Athens, Greece

49: Also at Riga Technical University, Riga, Latvia

50: Also at Universität Zürich, Zurich, Switzerland

51: Also at Stefan Meyer Institute for Subatomic Physics (SMI), Vienna, Austria

52: Also at Adiyaman University, Adiyaman, Turkey

53: Also at Istanbul Aydin University, Istanbul, Turkey

54: Also at Mersin University, Mersin, Turkey

55: Also at Piri Reis University, Istanbul, Turkey

56: Also at Izmir Institute of Technology, Izmir, Turkey

57: Also at Necmettin Erbakan University, Konya, Turkey

58: Also at Marmara University, Istanbul, Turkey

59: Also at Kafkas University, Kars, Turkey

60: Also at Istanbul Bilgi University, Istanbul, Turkey

61: Also at Rutherford Appleton Laboratory, Didcot, United Kingdom

62: Also at School of Physics and Astronomy, University of Southampton, Southampton, United Kingdom

63: Also at Monash University, Faculty of Science, Clayton, Australia

64: Also at Instituto de Astrofísica de Canarias, La Laguna, Spain

65: Also at Utah Valley University, Orem, U.S.A.

66: Also at Purdue University, West Lafayette, U.S.A.

67: Also at Beykent University, Istanbul, Turkey

68: Also at Bingol University, Bingol, Turkey

69: Also at Erzincan University, Erzincan, Turkey

70: Also at Sinop University, Sinop, Turkey

71: Also at Mimar Sinan University, Istanbul, Istanbul, Turkey

72: Also at Texas A\&M University at Qatar, Doha, Qatar

73: Also at Kyungpook National University, Daegu, Korea 